\title{
ON A CLASS OF NON-LINEAR FUNCTIONAL EQUATIONS CONNECTED WITH MODULAR FUNCTIONS
}

\author{
K. MAHLER \\ (Received 25 November 1974) \\ Communicated by Jennifer Seberry Wallis \\ Dedicated to George Szekeres on the occasion \\ of his 65th birthday
}

\section{Abstract}

Let $p$ be a prime. This paper deals with solutions of functional equations

$$
f\left(z^{p}\right)^{p+1}+f(z)^{p+1}+\sum_{r=0}^{p} \sum_{s=0}^{p} c_{r s} f\left(z^{p}\right)^{\prime} f(z)^{s}=0 \quad\left(c_{r s}=c_{s z}\right)
$$

in either formal Laurent series or in analytic functions. Examples connected to special modular functions are considered.

Many years ago, in several papers dealing mainly with questions of transcendency, I have studied (Mahler 1929, 1930a, 1930b) functional equations of the type

$$
f\left(z^{n}\right)=R(z, f(z))
$$

where $n \geqq 2$ is a fixed integer, and $R(z, w)$ is a rational function of its arguments the numerator and the denominator of which are at most of degree $n-1$ in $w$.

From the theory of transformation equations of modular functions one is led to study the related type of functional equations

$$
f\left(z^{n}\right)^{n+1}+f(z)^{n+1}+\sum_{r=0}^{n} \sum_{s=0}^{n} c_{r s} f\left(z^{n}\right)^{\prime} f(z)^{s}=0
$$

where the coefficients $c_{r s}$ are constants satisfying the symmetry conditions

$$
c_{r s}=c_{s r} \text { for all } r, s \text {. }
$$

Here the unknown function $f(z)$ is assumed to be analytic with at most a pole at $z=0$. 
Although this kind of functional equation can be studied for any integer $n \geqq 2$, the theory becomes particularly simple when $n=p$ is a prime. It is therefore this special class of functional equations which will be considered in the present paper. It would have some interest to investigate also the case of general $n$, and further to study the more general class of functional equations where the symmetry conditions (S) are not satisfied.

The first two chapters deal with solutions of the equation (E) that are formial Laurent series $f(z)$ in an indeterminate $z$, and we determine in particular the number of independent parameters on which the set of all possible solutions $f(z)$ of this kind may depend. The third chapter contains then a proof that the formal solutions $f(z)$ do in fact converge for $0<|z|<\gamma$ where $\gamma$ denotes a sufficiently small positive constant. This allows to prove that $f(z)$ may be continued into the whole unit circle, but may then possess an infinity of algebraic branch points.

In the last chapter we finally discuss connections of our results to special modular functions and some of their transformation equations. Here the group properties of the modular functions play only a very implicit role.

1. The basic modular function $j(\omega)$ of level 1 is invariant under the full modular group of all linear substitutions

$$
\omega \rightarrow \frac{\alpha \omega+\beta}{\gamma \omega+\delta} \quad(\alpha, \beta, \gamma, \delta \text { integers satisfying } \alpha \delta-\beta \gamma=1) .
$$

The function $j(\omega)$ is regular in the upper halfplane $\operatorname{Im}(\omega)>0$ and has a simple pole at infinity which is made evident by the convergent Fourier expansion

$$
j(\omega)=\sum_{h=-1}^{\infty} a_{h} e^{2 \pi i h \omega} \text { where } a_{-1}=1, a_{0}=744 \text {, etc. }
$$

Of fundamental importance are the transformation equations for $j(\omega)$. Let $n \geqq 2$ be any integer. There exists then a unique irreducible symmetric polynomial

$$
F_{n}(X, Y)=F_{n}(Y, X)
$$

of the exact degree

$$
\psi(n)=n \prod_{p \mid n}\left(1+\frac{1}{p}\right)
$$

in both $X$ and $Y$, with the highest terms

$$
X^{\psi(n)} \text { and } Y^{\psi(n)} \text {, }
$$

such that 


$$
F_{n}(j(n \omega), j(\omega)) \equiv 0 \text { identically in } \omega .
$$

More exactly, if $\{A, B, D\}$ runs over all $\psi(n)$ triplets of integers satisfying

$$
A>0, \quad 0 \leqq B \leqq D-1, \quad D>0, \quad A D=n, \quad(A, B, D)=1,
$$

then the equation

$$
F_{n}(X, j(\omega))=0
$$

has as its roots exactly all the $\psi(n)$ transformed functions

$$
j\left(\frac{A \omega+B}{D}\right)
$$

Of particular interest for us is the special case when $n=p$ is a prime and therefore $\psi(p)=p+1$. Now the transformation equation

$$
F_{p}(X, j(\omega))=0
$$

has the $p+1$ roots

$$
j(p \omega), j\left(\frac{\omega}{p}\right), j\left(\frac{\omega+1}{p}\right), \cdots, j\left(\frac{\omega+p-1}{p}\right),
$$

and the transformation polynomial $F_{p}(X, Y)$ has the explicit form

$$
F_{p}(X, Y)=-\left(X^{p}-Y\right)\left(Y^{p}-X\right)+\sum_{r=0}^{p} \sum_{s=0}^{p} c_{r s} X^{r} Y^{s}
$$

where the coefficients $c_{r s}$ are certain integers divisible by $p$ and satisfying

$$
c_{r s}=c_{s r}, \quad c_{p p}=0 .
$$

2. Put

$$
z=e^{2 \pi i \omega} \text { and } \quad j(\omega)=f(z)
$$

so that

$$
j(\omega)=f(z)=\sum_{h=-1}^{\infty} a_{h} z^{h}
$$

becomes a Laurent series in the integral powers of $z$ which converges for

$$
0<|z|<1 \text {. }
$$

(The new variable $z$ corresponds to the quantities $q^{2}$ of Jacobi and $r$ of Klein.) In terms of $f(z)$, the roots of the transformation equation 


$$
F_{p}(X, f(z))=0
$$

can be written as

$$
f\left(z^{p}\right), f\left(z^{1 / p}\right), f\left(\varepsilon z^{1 / p}\right), \cdots, f\left(\varepsilon^{p-1} z^{1 / p}\right)
$$

where

$$
\varepsilon=e^{2 \pi i / p}
$$

is a primitive $p$ th root of unity.

If $F_{p}(X, f(z))$ is written in its explicit form as a polynomial in $X$,

$$
F_{p}(X, f(z))=X^{p+1}-s_{1} X^{p}+s_{2} X^{p-1}-+\cdots \pm s_{p+1},
$$

then the coefficients $s_{1}, s_{2}, \cdots, s_{p+1}$ are the elementary symmetric functions of its roots,

$$
\begin{aligned}
& s_{1}=f\left(z^{p}\right)+f\left(z^{1 / p}\right)+f\left(\varepsilon z^{1 / p}\right)+\cdots+f\left(\varepsilon^{p-1} z^{1 / p}\right) \\
& s_{2}=f\left(z^{p}\right) f\left(z^{1 / p}\right)+f\left(z^{p}\right) f\left(\varepsilon z^{1 / p}\right)+\cdots+f\left(\varepsilon^{p-2} z^{1 / p}\right) f\left(\varepsilon^{p-1} z^{1 / p}\right) \\
& \cdot \\
& s_{p+1}=f\left(z^{p}\right) f\left(z^{1 / p}\right) f\left(\varepsilon z^{1 / p}\right) \cdots f\left(\varepsilon^{p-1} z^{1 / p}\right) .
\end{aligned}
$$

(1)

Each of the elementary symmetric functions is a polynomial in $f(z)$ with constant (integral) coefficients, and while $s_{1}, s_{2}, \cdots, s_{p}$ are at most of degree $p$ in $f(z), s_{p+1}$ has the exact degree $p+1$ in $f(z)$.

3. The properties just quoted have their analogues for other modular functions, including those of higher level. In this paper, we shall try to extend several of these properties to the solutions of a more general class of functional equations

$$
F\left(f\left(z^{p}\right), f(z)\right)=0
$$

where $F(X, Y)$ is a symmetric polynomial in $X$ and $Y$ of degree $p+1$. The more general kind of functional equation

$$
F\left(f\left(z^{n}\right), f(z)\right)=0
$$

where $n \geqq 2$ is a composite number, can be treated similarly, but requires the discussion of more cases. 


\section{Chapter 1. Formal Laurent Series}

4. For the present we shall not be concerned with analytic functions of $\omega$ or $z$, but rather with formal Laurent series in one indeterminate $z$.

Denote by $p$ a fixed prime and by $K$ an arbitrary field not of characteristic $p$ which contains a root $\varepsilon \neq 1$ of the equation $x^{p}-1=0$; its $p$ roots $1, \varepsilon, \varepsilon^{2}, \cdots, \varepsilon^{p-1}$ are then all distinct. Elements of $K$ will be called constants.

Next let $z$ be an indeterminate over $K$, and let $K[[z]]$ denote the field of all formal ascending Laurent series

$$
f(z)=\sum_{n=m}^{\infty} a_{n} z^{n}
$$

with coefficients $a_{h}$ in $K$. If the trivial case $f(z) \equiv 0$ is excluded, the notation can always be chosen such that $a_{m} \neq 0$. Then $m$ is called the order of $f(z)$; this order may be negative, zero or positive.

With the series $f(z)$ there can be associated the set, $\Sigma_{f}$ say, of the $p+1$ derived Laurent series

$$
\Sigma_{f}: \quad f\left(z^{p}\right) ; f\left(z^{1 / p}\right), f\left(\varepsilon z^{1 / p}\right), f\left(\varepsilon^{2} z^{1 / p}\right), \cdots, f\left(\varepsilon^{p-1} z^{1 / p}\right)
$$

in $z^{p}$, and $z^{1 / p}$, respectively, and we can further form the elementary symmetric functions $s_{1}, s_{2}, \cdots, s_{p+1}$ of these series, as defined in (1).

We now say that $f(z)$ is an $S_{p}$-series if

(A) No two of the elements of $\Sigma_{f}$ are identical; and

(B) Every elementary symmetric function $s_{1}, s_{2}, \cdots, s_{p+1}$ of the elements of $\Sigma_{f}$ can be expressed as a polynomial in $f(z)$ with coefficients in $K$.

5. A number of simple properties of such $S_{p}$-series $f(z)$ follow immediately from this definition.

Firstly, by (A), $f(z)$ cannot be a constant since then all the elements of $\Sigma_{f}$ would be equal to the same constant.

Secondly, since then $f(z) \notin K$, it can easily be proved that $f(z)$ is transcendental over $K$.

Thirdly, if $C_{0} \neq 0$ and $C_{1}$ are arbitrary constants, also $C_{0} f(z)+C_{1}$ is an $S_{p}$-series and this series has the same order as $f(z)$, unless $f(z)$ has the order 0 .

Fourthly, if $n$ is any positive integer, also $f\left(z^{n}\right)$ is an $S_{p}$-series, but of order $m n$.

Fifth, the representations of the elementary functions $s_{k}$ as polynomials in $f(z)$ with coefficients in $K$ are unique by the transcendency of $f(z)$.

The last property implies that we can associate with every $S_{p}$-series $f(z)$ a 
unique polynomial $F(X, Y)$ in two independent indeterminates $X$ and $Y$ by putting

$$
\begin{aligned}
F(X, f(z)) & =\left(X-f\left(z^{p}\right)\right) \prod_{i=0}^{p-1}\left(X-f\left(\varepsilon^{i} z^{1 / p}\right)\right) \\
& =X^{p+1}-s_{1} X^{p}+s_{2} X^{p-1}-+\cdots \pm s_{p+1} .
\end{aligned}
$$

This polynomial $F(X, Y)$ has again coefficients in $K$; we call it the polynomial of $f(z)$. Before studying this polynomial, we establish a general property of certain symmetric polynomials.

6. Denote by $\Phi(X, Y)=\Phi(Y, X)$ any symmetric polynomial in $X$ and $Y$ of the form

$$
\Phi(X, Y)=X^{p+1}+Y^{p+1}+\sum_{r=0}^{p} \sum_{s=0}^{p} \gamma_{r s} X^{r} Y^{s}
$$

with coefficients $\gamma_{r s}=\gamma_{s r}$ in $K$. The following result can then be proved.

THEOREM 1. Let $f(z)$ be a series in $K[[z]]$ such that all the elements of $\Sigma_{1}$ are distinct. If any one of the $p+1$ equations

$\Phi\left(f\left(z^{p}\right), f(z)\right)=0$ and $\Phi\left(f\left(\varepsilon^{j} z^{1 / p}\right), f(z)\right)=0$, where $j=0,1, \cdots, p-1$, is satisfied, then $f(z)$ is an $S_{p}$-series and $\Phi(X, Y)$ is its polynomial.

Proof. It evidently suffices to prove that each of these equations implies the other $p$ equations; for then the elementary symmetric functions $s_{k}$ of the elements of $\Sigma_{f}$ become polynomials in $f(z)$ with coefficients in $K$ since they are the roots of the monic equation

$$
\Phi(X, f(z))=0 .
$$

If, firstly,

$$
\Phi\left(f\left(z^{p}\right), f(z)\right)=0,
$$

then, on replacing $z$ by $\varepsilon^{i} z^{1 / p}$, it follows that also

$$
\Phi\left(f(z), f\left(\varepsilon^{j} z^{1 / p}\right)\right)=0 \quad(j=0,1, \cdots, p-1),
$$

and the assertion is an immediate consequence of the symmetry of $\Phi(X, Y)$.

Secondly, let for some $j$

$$
\Phi\left(f\left(\varepsilon^{i} z^{1 / p}\right), f(z)\right)=0 .
$$

If now $\varepsilon^{j} z^{1 / p}$ is replaced by $z$, and hence $\left(\varepsilon^{j} z^{1 / p}\right)^{p}=z$ by $z^{p}$, we obtain the equation 


$$
\Phi\left(f(z), f\left(z^{p}\right)\right)=0,
$$

and so, by the symmetry of $\Phi(X, Y)$, we are back in the first case in which the assertion has already been proved.

7. The following theorem is basic for the further theory.

THEOREM 2. Let $f(z)$ be any $S_{p}$-series, and let $F(X, Y)$ be the polynomial of $f(z)$. Then this polynomial is symmetric in $X$ and $Y$ and hence is of the form

$$
F(X, Y)=X^{p+1}+Y^{p+1}+\sum_{r=0}^{p} \sum_{s=0}^{p} c_{r s} X^{r} Y^{s}
$$

with coefficients $c_{r s}$ in $K$ satisfying the symmetry conditions

$$
c_{r s}=c_{s r} \quad(r, s=1, \cdots, p) .
$$

Proof. Since $F(X, Y)$ is monic in $S$, it suffices to prove the symmetry of $F(X, Y)$ because it has then the asserted form and the coefficients $c_{r s}$ satisfy $c_{r s}=c_{s r}$.

Two cases have to be distinguished. First assume that $F(X, Y)$ is irreducible over $K$. Since both $f\left(z^{p}\right)$ and $f\left(z^{1 / p}\right)$ lie in $\Sigma_{f}$, evidently

$$
F\left(f\left(z^{p}\right), f(z)\right)=0 \quad \text { and } \quad F\left(f\left(z^{1 / p}\right), f(z)\right)=0 .
$$

On replacing $z$ by $z^{p}$ in the second equation, it follows that also

$$
F\left(f(z), f\left(z^{p}\right)\right)=0 .
$$

The irreducible equation

$$
F(X, f(z))=0
$$

and the equation

$$
F(f(z), X)=0
$$

have therefore the root $f\left(z^{p}\right)$ in common and therefore share all the $p+1$ elements of $\Sigma_{f}$ as roots. This requires, firstly, that $F(X, Y)$ is at least of degree $p+1$ in $Y$. Secondly, $F(Y, X)$ necessarily is divisible by $F(X, Y)$ and hence has the form

$$
F(Y, X)=h(X, Y) F(X, Y)
$$

where $h(X, Y)$ is a certain polynomial in $K[X, Y]$. Here the degree in $Y$ of $F(Y, X)$ is exactly $p+1$, and that of $F(X, Y)$ is at least $p+1$. Hence both polynomials are exactly of the same degrees $p+1$ in both $X$ and $Y$, and so the factor 


$$
h(X, Y)=h
$$

is a constant. On applying twice the identity (5), once after interchanging $X$ and $Y$, it follows that

$$
F(Y, X)=h F(X, Y)=h^{2} F(Y, X),
$$

hence that $h^{2}=1$. Here $h$ cannot have the value -1 since then $F(Y, X)$ $=-F(X, Y)$ and therefore $F(X, X) \equiv 0$ so that $F(X, Y)$ is divisible by $X-Y$, contrary to the irreducibility of $F(X, Y)$. Hence $h=+1$ and $F(X, Y)$ $=F(Y, X)$, whence the assertion.

Secondly let $F(X, Y)$ be reducible. It has then a factorisation

$$
F(X, Y)=G(X, Y) H(X, Y)
$$

where both $G(X, Y)$ and $H(X, Y)$ are polynomials in $K[X, Y]$ which have positive degrees in $X$. The two equations

$$
G(X, f(z))=0
$$

and

$$
H(X, f(z))=0
$$

together have all the $p+1$ elements of $\Sigma_{f}$ as their roots. Without loss of generality the notation can be chosen such that the first equation (6) is irreducible and has $f\left(z^{1 / p}\right)$ as one of its roots. Replace $z^{1 / p}$ in this equation (6) successively by $\varepsilon z^{1 / p}, \varepsilon^{2} z^{1 / p}, \cdots, \varepsilon^{p-1} z^{1 / p}$. These substitutions leave both $z$ and $f(z)$ unchanged, but transform $f\left(z^{1 / p}\right)$ into $f\left(\varepsilon z^{1 / p}\right), f\left(\varepsilon^{2} z^{1 / p}\right), \cdots, f\left(\varepsilon^{p-1} z^{1 / p}\right)$, respectively, which therefore likewise satisfy the equation (6). This equation cannot have more than $p$ roots, and so the remaining element $f\left(z^{p}\right)$ of $\Sigma_{f}$ necessarily satisfies the second equation (7). This equation (7) is linear and so is also irreducible.

It follows then that

$$
G\left(f\left(z^{1 / p}\right), f(z)\right)=0 \quad \text { and } \quad H\left(f\left(z^{p}\right), f(z)\right)=0,
$$

hence, on replacing $z$ by $z^{p}$ and $z^{1 / p}$, respectively, that also

$$
G\left(f(z), f\left(z^{p}\right)\right)=0 \text { and } H\left(f(z), f\left(z^{1 / p}\right)\right)=0 .
$$

It has thus been shown that $f\left(z^{1 / p}\right)$ is a root of both irreducible equation (6) and of the equation

$$
H(f(z), X)=0,
$$

and similarly that $f\left(z^{p}\right)$ is a root of both the irreducible equation (7) and of

$$
G(f(z), X)=0 .
$$


These properties require that $H(Y, X)$ be divisible by $G(X, Y)$ and $G(Y, X)$ be divisible by $H(X, Y)$, hence that $G(X, Y)$ also be divisible by $H(Y, X)$. Hence there is a constant $h \neq 0$ such that

$$
G(X, Y)=h H(Y, X)
$$

and therefore that

$$
F(X, Y)=G(X, Y) H(X, Y)=h H(X, Y) H(Y, X) .
$$

This identity shows that also in the present case $F(X, Y)$ is symmetrical in $X$ and $Y$.

8. Let again $f(z)$ be an $S_{p}$-series, and let

$$
F(X, Y)=X^{p+1}+Y^{p+1}+\sum_{r=0}^{p} \sum_{s=0}^{p} c_{r s} X^{r} Y^{s} \quad\left(c_{r s}=c_{s r}\right)
$$

be its polynomial. We know already that if $C_{0} \neq 0$ and $C_{1}$ are arbitrary constants, then also

$$
g(z)=C_{0} f(z)+C_{1}
$$

is an $S_{p}$-series. Denote by

$$
G(X, Y)=X^{p+1}+Y^{p+1}+\sum_{r=0}^{p} \sum_{s=0}^{p} d_{r s} X^{r} Y^{s}, \quad\left(d_{r s}=d_{s r}\right)
$$

the polynomial of $g(z)$.

The set $\Sigma_{\mathrm{g}}$ consists of the $p+1$ Laurent series

$$
\begin{aligned}
g\left(z^{p}\right) & =C_{0} f\left(z^{p}\right)+C_{1} \\
g\left(\varepsilon^{\prime} z^{1 / p}\right) & =C_{0} f\left(\varepsilon^{\prime} z^{1 / p}\right)+C_{1} \quad \text { where } j=0,1, \cdots, p-1,
\end{aligned}
$$

and these series are all distinct and satisfy the equation

$$
G(X, g(z))=0,
$$

while similarly the elements of $\Sigma_{f}$ are the roots of the equation

$$
F(X, f(z))=0 .
$$

Here both polynomials $F(X, Y)$ and $G(X, Y)$ are monic with respect to $X$ and to $Y$. They are therefore connected by the identity

$$
C_{0}^{p+1} G(X, Y)=F\left(C_{0} X+C_{1}, C_{0} Y+C_{1}\right),
$$

from which it easily follows that 


$$
C_{0}^{p+1} d_{00}=2 C_{1}^{p+1}+\sum_{R=0}^{p} \sum_{S=0}^{p} c_{R S} C_{1}^{R+s},
$$

$$
C_{0}^{p-s+1} d_{0 s}=\left(\begin{array}{c}
p+1 \\
s
\end{array}\right) C_{1}^{p-s+1}+\sum_{R=0}^{p} \sum_{S=s}^{p} c_{R S}\left(\begin{array}{l}
S \\
s
\end{array}\right) C_{1}^{R+s-s} \quad(s=1,2, \cdots, p),
$$

$$
C_{0}^{p-r-s+1} d_{r s}=\sum_{R=r}^{p} \sum_{S=s}^{p} c_{R S}\left(\begin{array}{l}
R \\
r
\end{array}\right)\left(\begin{array}{l}
S \\
s
\end{array}\right) C_{1}^{R+s-r-s} \quad(r, s=1,2, \cdots, p) .
$$

In the special case when $C_{1}=0$ and therefore

$$
g(z)=C_{0} f(z),
$$

these formulae take the simpler form

$$
d_{r s}=C_{0}^{r+s-p-1} c_{r s} \quad(r, s=0,1, \cdots, p) .
$$

We further note that, under no restriction on $C_{1}$, the equations (9c) imply in particular that

$$
d_{p p}=C_{0}^{p-1} c_{p p}
$$

9. There is one further set of transformation formulae which will soon be needed.

Let again $f(z)$ be any $S_{p}$-series and let $F(X, Y)$ be its polynomial. We say that $f(z)$ is an $R_{p}$-series if also its reciprocal $f(z)^{-1}$ is an $S_{p}$-series. Thus both $f(z)$ and $f(z)^{-1}$ are simultaneously $S_{p}$-series and $R_{p}$-series.

Assume that $f(z)$ is such an $R_{p}$-series, and denote as before by $F(X, Y)$ the polynomial of $f(z)$ and similarly by

$$
F^{0}(X, Y)=X^{p+1}+Y^{p+1}+\sum_{r=0}^{p} \sum_{s=0}^{p} c_{r s}^{0} X^{\prime} Y^{s} \quad\left(c_{r s}^{0}=c_{s r}^{0}\right)
$$

the polynomial of $f(z)^{-1}$. The set $\Sigma_{f^{-1}}$ consists of the $p+1$ Laurent series

$$
f\left(z^{p}\right)^{-1} ; f\left(z^{1 / p}\right)^{-1}, f\left(\varepsilon z^{1 / p}\right)^{-1}, \cdots, f\left(\varepsilon^{p-1} z^{1 / p}\right)^{-1},
$$

and both $F(X, Y)$ and $F^{0}(X, Y)$ are monic and of the exact degrees $p+1$ in $X$ and in $Y$. These two polynomials are therefore connected by the pair of identities

$$
F^{0}(X, Y)=X^{p+1} Y^{p+1} F\left(X^{-1}, Y^{-1}\right), \quad F(X, Y)=X^{p+1} Y^{p+1} F^{0}\left(X^{-1}, Y^{-1}\right) .
$$

The explicit formula (3) for $F(X, Y)$ implies then that $F^{0}(X, Y)$ is given by

$$
F^{0}(X, Y)=X^{p+1}+Y^{p+1}+\sum_{r=0}^{p} \sum_{s=0}^{p} c_{r s} X^{p-r+1} Y^{p-s+1},
$$


ind here both exponents $p-r+1$ and $p-s+1$ are at least 1 . But $F^{0}(X, Y)$ may tot contain any terms divisible by $X^{p+1} Y$ or by $X Y^{p+1}$. Therefore necessarily

$$
c_{0 s}=c_{s 0}=0 \quad(s=0,1, \cdots, p) .
$$

Hence in the present case $F(X, Y)$ has the special form

$$
F(X, Y)=X^{p+1}+Y^{p+1}+\sum_{r=1}^{p} \sum_{s=1}^{p} c_{r s} X^{r} Y^{s}
$$

and similarly $F^{0}(X, Y)$ has the form

$$
F^{0}(X, Y)=X^{p+1}+Y^{p+1}+\sum_{r=1}^{p} \sum_{s=1}^{p} c_{r s}^{0} X^{r} Y^{s}
$$

with the coefficients

$$
c_{r s}^{0}=c_{p-r+1, p-s+1} \quad(r, s=1,2, \cdots, p) .
$$

By the complete symmetry in $F(X, Y)$ and $F^{0}(X, Y)$ we can then conclude that

The conditions (13) are both necessary and sufficient for $f(z)$ to be an $R_{p}$-series.

It is useful to note that both the double sums $\sum_{r=1}^{p} \sum_{s=1}^{p}$ in (14a) and (14b) are tivisible by $X Y$, hence are equal to zero if $X=0$ or $Y=0$.

10. The following theorem shows the special role of series of positive order.

THEOREM 3. Every $S_{p}$-series of positive order is also an $R_{p}$-series.

Proof. Let $f(z)$ be any $S_{p}$-series of positive order. It suffices to show that he polynomial $F(X, Y)$ of $f(z)$ has the form (14a), i.e. that its coefficients $c_{r s}$ ;atisfy the conditions (13).

Assume this assertion is not true. There exists then a suffix $\sigma$ in the interval ) $\leqq \leqq p$ such that

$$
c_{0 s}=0 \text { for } s=0,1, \cdots, \sigma-1 \text {, but that } c_{0 \sigma} \neq 0 .
$$

We apply the identity

$$
F\left(f\left(z^{p}\right), f(z)\right)=f\left(z^{p}\right)^{p+1}+f(z)^{p+1}+\sum_{r=0}^{p} \sum_{s=0}^{p} c_{r s} f\left(z^{p}\right)^{\prime} f(z)^{s}=0 .
$$

Here by hypothesis

$$
f(z)=a_{m} z^{m}+\cdots \text { and } f\left(z^{p}\right)=a_{m} z^{m p}+\cdots
$$

where $m>0, a_{m} \neq 0$, and the dots denote terms in higher powers of $z$. The identity implies then that 


$$
a_{m}^{p+1} z^{m\left(p^{2}+p\right)}+\cdots+a_{m}^{p+1} z^{m(p+1)}+\cdots+\sum_{r=0}^{p} \sum_{s=0}^{p} c_{r s}\left(a_{m}^{r+s} z^{m(p r+s)}+\cdots\right)=0 .
$$

If now $0 \leqq \sigma \leqq p-1$, then there is in this identity only one term $c_{0 \sigma} a_{m}^{\sigma} z^{m \sigma}$ involving the lowest occurring power of $z$, and this term cannot be cancelled by any other term. If, however, $\sigma=p$, then $c_{10}=c_{01}=0$ by the definition of $\sigma$, and so $c_{0 p} a_{m}^{p} z^{m p}$, which is now the term involving the lowest power of $z$, again cannot be cancelled by any other term. This proves the assertion.

We shall soon show that there are $S_{p}$-series of negative order which are not also $R_{p}$-series. There is thus an essential difference between the $S_{p}$-series of positive and of negative orders.

11. The next problem to be discussed concerns the question whether, for a given integer $m$ and a given constant $a_{m} \neq 0$, there exists an $S_{p}$-series

$$
f(z)=\sum_{h=m}^{\infty} a_{h} z^{h}
$$

of order $m$ and with the first coefficient $a_{m}$. The answer will depend on whether $m$ is positive, zero, or negative, and the conditions will be different in these three cases.

We begin with the easiest case when $m$ is negative, and we apply again the identity (16) where now, however, $m$ is a negative integer.

On writing the double sum in this identity as a Laurent series, the term in the highest occurring negative power of $z$ evidently is

$$
c_{p p} a_{m}^{2 p} \cdot z^{m\left(p^{2}+p\right)} \text {. }
$$

Similarly, the term in the highest occurring negative power of $z$ arising from

$$
\left(a_{m} z^{m p}+\cdots\right)^{p+1}+\left(a_{m} z^{m}+\cdots\right)^{p+1}
$$

is equal to

$$
a_{m}^{p+1} \cdot z^{m\left(p^{2}+p\right)} .
$$

These two terms must cancel one another, whence there follows the necessary condition

$$
c_{p p}=-a_{m}^{-p+1}
$$

for $a_{m}$. This equation shows in particular that

the coefficient $c_{p p}$ of the polynomial $F(X, Y)$ of $f(z)$ is distinct from zero if $m<0$, and $-c_{p p}$ is then the $(p-1)$ st power of an element of $K$.

12. In order to derive also sufficient conditions, it is convenient to put 


$$
f(z)=a_{m} g(z), \text { where } g(z)=z^{m}+\sum_{n=m+1}^{\infty} b_{h} z^{n}
$$

is again an $S_{\mathrm{p}}$-series of order $m<0$, but with the special property that 1 is the coefficient of $z^{m}$. Such a series is said to be normed.

As in $\$ 8$, denote by

$$
G(X, Y)=X^{p+1}+Y^{p+1}+\sum_{r=0}^{p} \sum_{s=0}^{p} d_{r s} X^{r} Y^{s} \quad\left(d_{r s}=d_{s r}\right)
$$

the polynomial of $g(z)$. By (10), this polynomial has the coefficients

$$
d_{r s}=a_{m}^{r+s-p-1} c_{r s} \quad(r, s=0,1, \cdots, p) .
$$

In particular, the necessary condition (17) takes now the simple form

$$
d_{p p}=-1 \text {. }
$$

This formula suggests to write $G(X, Y)$ in the equivalent, but more. convenient form

$$
G(X, Y)=-\left(X^{p}-Y\right)\left(Y^{p}-X\right)+\sum_{r=0}^{p} \sum_{s=0}^{p} D_{r s} X^{r} Y^{s} \quad\left(D_{r s}=D_{s r}\right)
$$

where

$$
\begin{aligned}
D_{p p}=d_{p p}+1=0, \quad D_{11}=d_{11}+1, \quad \text { and otherwise } & D_{r s}=d_{r s} \\
& (r, s=0,1, \cdots, p) .
\end{aligned}
$$

Denote now by $D$ the set of all coefficients

$$
D_{r s}, \text { where } 0 \leqq r \leqq s \leqq p,
$$

with the exception of the trivial coefficient $D_{p p}=0$. Further, if $n$ is any integer greater than $m$, let $B_{n}$ be any polynomial in

$$
b_{m+1}, b_{m+2}, \cdots, b_{n} \text { and in the elements of } D
$$

with coefficients in $K$. The symbol $B_{n}$ need not always denote the same polynomial.

13. The following basic existence result can now be proved.

THEOREM 4. Let $G(X, Y)$ be any symmetric polynomial of the form (20) with coefficients $D_{r s}$ in $K$ where $D_{p p}=0$, and let $m$ be any negative integer. Then there exists one and only one normed $S_{p}$-series $g(z)$ of order $m$ such that $G(X, Y)$ is its polynomial. 
Proof. In analogy to earlier proofs, we apply the identity

$$
\begin{aligned}
& G\left(g\left(z^{p}\right), g(z)\right)= \\
& \quad=\left(g\left(z^{p}\right)^{p}-g(z)\right)\left(g(z)^{p}-g\left(z^{p}\right)\right)+\sum_{r=0}^{p} \sum_{s=0}^{p} D_{r s} g\left(z^{p}\right)^{r} g(z)^{s}=0 .
\end{aligned}
$$

Here

$$
g(z)=z^{m}+\sum_{h=1}^{\infty} b_{h+m} z^{h+m}, \quad g\left(z^{p}\right)=z^{m p}+\sum_{h=1}^{\infty} b_{h+m} z^{(h+m) p},
$$

hence

$$
\begin{gathered}
g(z)^{s}=z^{m s}+s b_{m+1} z^{m s+1}+\sum_{h=2}^{\infty}\left(s b_{h+m}+B_{h+m-1}\right) z^{m s+h}, \\
g\left(z^{p}\right)^{r}=z^{m p r}+r b_{m+1} z^{(m s+1) p}+\sum_{h=2}^{\infty}\left(r b_{h+m}+B_{h+m-1}\right) z^{(m r+h) p} .
\end{gathered}
$$

Further, since $p$ is at least 2 ,

$$
g\left(z^{p}\right)^{p}-g(z)=z^{m p^{2}}+\sum_{h=2}^{\infty} B_{h+m-1} z^{m p^{2}+h}
$$

and

$$
g(z)^{p}-g\left(z^{p}\right)=p b_{m+1} z^{p m+1}+\sum_{h=2}^{\infty}\left(p b_{h+m}+B_{h+m-1}\right) z^{m p+h}
$$

whence

$$
\begin{aligned}
& \left(g\left(z^{p}\right)^{p}-g(z)\right)\left(g(z)^{p}-g(z)\right)= \\
& \quad=p b_{m+1} z^{m\left(p^{2}+p\right)+1}+\sum_{h=2}^{\infty}\left(p b_{h+m}+B_{h+m-1}\right) z^{m\left(p^{2}+p\right)+h} .
\end{aligned}
$$

On the other hand,

$$
\begin{aligned}
D_{r s} g\left(z^{p}\right)^{r} g(z)^{s}=D_{r s} z^{m(p r+s)}+\sum_{h=1}^{\infty} B_{h+m} z^{m(p r+s)+h} & \\
& (r, s=0,1, \cdots, p) .
\end{aligned}
$$

On substituting these Laurent series in (22) and equating the coefficients of all the different powers of $z$ to zero, we obtain an infinite system of recursive formulae

$$
p b_{m+1}=D_{p, p-1} ; \quad p b_{h+m}=B_{h+m-1} \quad \text { for } \quad h=2,3,4, \cdots
$$


These formulae allow to determine step by step all the coefficients $b_{h+m}$ and prove both their existence and uniqueness.

The proof implies the following result.

COROLlaRY. The coefficients $b_{h+m}$ of $g(z)$ can be expressed as polynomials in the elements of $D$ with coefficients in $K$.

By means of a slight change of method one can show that, conversely, all the elements of $D$ can be written as polynomials in finitely many of the Laurent coefficients $b_{h+m}$ with coefficients in $K$. This is also implicit in the definition of $G(X, Y)$ by means of the elementary symmetric functions of the elements of $\Sigma_{g}$.

14. Denote by $g(z \mid m)$ the normed $S_{p}$-series of order $m<0$ given by Theorem 4, and put

$$
g[z]=g(z \mid-1)
$$

The identity

$$
G\left(g\left[z^{p}\right], g[z]\right)=0
$$

implies that for every positive integer $n$ also

$$
G\left(g\left[z^{n p}\right], g\left[z^{n}\right]\right)=0,
$$

and here, by $\S 5, g\left[z^{n}\right]$ is again a normed $S_{p}$-series, but of order $-n$. The uniqueness proved in Theorem 4 implies then the identity

$$
g(z \mid m)=g\left[z^{-m}\right] \quad \text { for every integer } m<0 .
$$

Thus, once $g[z]$ is known, we know all the normed $S_{p}$-series of negative orders of which $G(X, Y)$ is the polynomial.

On returning to the original $S_{p}$-series $f(z)$ of order $m<0$ with coefficient $a_{m} \neq 0$, Theorem 4 leads easily to the following more general result.

THEOREM 5. Let

$$
F(X, Y)=X^{p+1}+Y^{p+1}+\sum_{r=0}^{p} \sum_{s=0}^{p} c_{r s} X^{r} Y^{s}
$$

be any symmetric polynomial with coefficients $c_{r s}$ in $K$ where $c_{p p} \neq 0$, and let

$$
f(z)=\sum_{n=m}^{\infty} a_{h} z^{n}
$$

be any $S_{p}$-series of order $m<0$ with the polynomial $F(X, Y)$. Then $a_{m}$ necessarily is a root in $K$ of the algebraic equation 


$$
a_{m}^{p-1}=-c_{p p}^{-1}
$$

and $f(z)$ has the form

$$
f(z)=a_{m} \cdot g\left[z^{-m}\right]
$$

where $g[z]$ is the unique normed $S_{p}$-series of order -1 with the polynomial

$$
G(X, Y)=a_{m}^{-(p+1)} F\left(a_{m} X, a_{m} Y\right) \text {. }
$$

This result shows that it suffices to determine all normed $S_{p}$-series of order -1 in order to obtain all $S_{p}$-series of arbitrary negative order.

15. The symmetric polynomial $F(X, Y)$ contains $P=[(p+1)(p+2)] / 2$ essential coefficients, i.e., coefficients $c_{r s}$ for which $0 \leqq r \leqq s \leqq p$. The most general $S_{p}$-series $f(z)$ of negative order depends therefore on $P$ independent parameters $c_{r s}$ in $K$ and in addition on the negative integer $m$ which is its order. On the other hand, the normed $S_{p}$-series $g[z]$ of order -1 involves only $P-1$ parameters for which we may take the elements of the set $D$ defined in $\$ 12$.

A further rather trivial reduction is possible. In the development

$$
g[z]=z^{-1}+\sum_{h=0}^{\infty} b_{h} z^{h}
$$

the constant term $b_{0}$ plays no essential role, and we may consider the $S_{p}$-series

$$
h[z]=g[z]-a_{0}=z^{-1}+\sum_{h=1}^{\infty} b_{h} z^{h}
$$

instead of $g[z]$. Also $h[z]$ is a normed $S_{p}$-series of order -1 ; we call it a basic $S_{p}$-series. Let

$$
H(X, Y)=-\left(X^{p}-Y\right)\left(Y^{p}-X\right)+\sum_{r=0}^{p} \sum_{s=0}^{p} E_{r s} X^{r} Y^{s} \quad\left(E_{r s}=E_{s r}\right)
$$

be the polynomial of $h[z]$. By the proof in $\S 12$,

$$
E_{p p}=0 \text {. }
$$

To this condition we can add the further one,

$$
E_{p-1, p}=E_{p, p-1}=0 \text {. }
$$

It can easily be proved by evaluating the coefficient of the power

$$
z^{-p^{2}-p+1}
$$

in the identity

$$
E\left(h\left[z^{p}\right], h[z]\right)=0
$$


It follows that the set of all basic $S_{p}$-series depends on only

$$
P-2=\frac{p(p+3)}{2}
$$

independent parameters in $K$, viz., on the coefficients

$$
E_{r s}, \quad \text { where } 0 \leqq r \leqq s \leqq p,
$$

Jut with the exclusion of the two trivial coefficients $E_{p p}=0$ and $E_{p-1, p}=0$.

We also see that, on allowing $C_{0} \neq 0$ and $C_{1}$ to run over all constants, $m$ to un over all negative integers, and $h[z]$ to run over all basic $S_{p}$-series, the ixpression

$$
f(z)=C_{0} h\left[z^{-m}\right]+C_{1}
$$

lescribes all $S_{p}$-series of negative orders.

16. Theorem 3 showed that if $f(z)$ is an $S_{p}$-series of positive order $m$, then $f(z)^{-1}$ is an $S_{p}$-series of the negative order $-m$. We found that in this case the solynomial $F(X, Y)$ of $f(z)$ and the polynomial $F^{0}(X, Y)$ of $f(z)^{-1}$ had the forms (14a) and (14b) in $\$ 9$, respectively. Both these polynomials contained only

$$
Q=\frac{p(p+1)}{2}
$$

sssential coefficients on account of the conditions (13). If

$$
f(z)=\sum_{h=m}^{\infty} a_{h} z^{h}
$$

he reciprocal function $f(z)^{-1}$ has a Laurent series which begins with the term $a_{m}^{-1} z^{-m}$. Hence

$$
g(z)=a_{m} f(z)^{-1}
$$

s a normed $S_{p}$-series, and even, by Theorem 3, a normed $R_{p}$-series; it has the negative order $-m$. If the polynomial is written in the form

$$
G(X, Y)=-\left(X^{p}-Y\right)\left(Y^{p}-X\right)+\sum_{r=0}^{p} \sum_{s=0}^{p} D_{r s} X^{r} Y^{s} \quad\left(D_{r s}=D_{s r}\right),
$$

then $\$ 9$ and $\$ 12$ imply that

$$
D_{0 s}=D_{s 0}=0 \text { for } s=0,1, \cdots, p \text {, and } D_{p p}=0 .
$$

Here the first conditions hold because $g(z)$ is an $R_{p}$-series, and the last one since $\mathrm{g}(z)$ is normed. 
Conversely, by Theorem 4, these properties of the coefficients of $G(X, Y)$ show that there exists to every negative integer $-m$ one and only one normed $S_{p}$-series $g(z)$ of the negative order $-m$ belonging to $G(X, Y)$, and this series is an $R_{p}$-series.

Denote again by $g[z]$ the unique normed $S_{p}$-series of order -1 the polynomial of which is $G(X, Y)$. Then for every positive integer $m$ the normed $S_{p}$-series $g\left[z^{m}\right]$ is the unique $S_{p}$-series of the negative order $-m$ belonging to $G(X, Y)$. In terms of this series the original $S_{p}$-series $f(z)$ can be written as

$$
f(z)=a_{m} g\left[z^{m}\right]^{-1} .
$$

It has in the present case no advantage to express in this formula $g[z]$ by the corresponding basic series $h[z]$.

We had put

$$
Q=\frac{p(p+1)}{2}
$$

From the conditions for the coefficients $D_{r s}$ of $G(X, Y)$, the normed series $g[z]$ depends only on the $Q-1$ essential coefficients

$$
D_{r s}, \quad \text { where } 1 \leqq r \leqq s \leqq p,
$$

of $G(X, Y)$ where the trivial coefficient $D_{p p}=0$ has been excluded. The $Q-1$ essential coefficients of $G(X, Y)$ may run independently over $K$. Therefore the set of all $S_{p}$-series $f(z)$ of arbitrary positive orders depends on exactly $Q$ parameters in $K$ because $a_{m} \neq 0$ may still run over all constants. Naturally $f(z)$ in addition depends on its positive order $m$.

Since $P>Q$, the following result is obtained.

THEOREM 6. While the reciprocal of every $S_{p}$-series of positive order is again an $S_{p}$-series, but of negative order, the reciprocal of an $S_{p}$-series of negative order is not in general an $S_{p}$-series.

17. There remains the study of the $S_{p}$-series of order 0 . Let

$$
f(z)=\sum_{h=0}^{\infty} a_{h} z^{h}, \text { where } a_{0} \neq 0,
$$

be such a series. Since $f(z)$ may not be a constant, there exists a positive suffix $m$ such that

$$
a_{1}=a_{2}=\cdots=a_{m-1}=0 \text {, but } a_{m} \neq 0 .
$$


Hence

$$
f(z)=a_{0}+g(z)
$$

where

$$
g(z)=\sum_{h=m}^{\infty} a_{h} z^{h}
$$

is an $S_{p}$-series of the positive order $m$ and so, by Theorem 3 , is an $R_{p}$-series.

This implies, by $\S 9$, that the polynomial $G(X, Y)$ of $g(z)$ has the form

$$
G(X, Y)=X^{p+1}+Y^{p+1}+\sum_{r=1}^{p} \sum_{s=1}^{p} d_{r s} X^{r} Y^{s} \quad\left(d_{r s}=d_{s r}\right) .
$$

Let similarly

$$
F(X, Y)=X^{p+1}+Y^{p+1}+\sum_{r=0}^{p} \sum_{s=0}^{p} c_{r s} X^{r} Y^{s} \quad\left(c_{r s}=c_{s r}\right)
$$

be the polynomial of $f(z)$. Then, by the relation (30),

$$
F(X, Y)=G\left(X-a_{0}, Y-a_{0}\right)
$$

identically in $X$ and $Y$, a formula which defines the polynomial of every possible $S_{p}$-series of order 0 . We see that such a series $f(z)$ depends on altogether $Q+1$ parameters in $K$, namely the $Q$ essential coefficients of $G(X, Y)$ and the coefficient $a_{0} \neq 0$. In addition, $f(z)$ depends also on the arbitrary positive integer $m$, and it may be written in the form

$$
f(z)=a_{0}+a_{m} g\left[z^{m}\right]^{-1},
$$

where $g[z]^{-1}$ denotes the unique normed $S_{p}$-series of order 1 with the polynomial $G(X, Y)$.

18. As has just been shown, the set of all $S_{p}$-series of order 0 depends on $Q+1$ parameters in $K$. On the other hand, we proved in $\$ 16$ that the set of all $S_{\mathrm{p}}$-series of positive order involves only $Q$ such parameters, and it follows from $\$ \$ 9-10$ that the same is true for the set of all $R_{p}$-series.

Therefore, in general, an $S_{p}$-series $f(z)$ of order 0 is not an $R_{p}$-series and hence its reciprocal $f(z)^{-1}$ is not an $S_{p}$-series.

The question arises then whether there do exist exceptional $S_{p}$-series of order 0 which are $R_{p}$-series. This question can be answered as follows.

Let $f(z)$ be such an $R_{p}$-series of order 0 , and let again $g(z)$ be the series of positive order defined by the equation (30); it is of positive order and therefore is an $R_{p}$-series. Let $G(X, Y)$ be the polynomial of $g(z)$ as given in the last section, and let similarly $F(X, Y)$ be the polynomial of $f(z)$. This polynomial has now the simpler form 


$$
F(X, Y)=X^{p+1}+Y^{p+1}+\sum_{r=1}^{p} \sum_{s=1}^{p} c_{r s} X^{r} Y^{s} \quad\left(c_{r s}=c_{s r}\right)
$$

The identity (31) can also be written as $F\left(X+a_{0}, Y+a_{0}\right)=G(X, Y)$ and is equivalent to

$$
\begin{aligned}
\left(X+a_{0}\right)^{p+1}+ & \left(Y+a_{0}\right)^{p+1}+\sum_{r=1}^{p} \sum_{s=1}^{p} c_{r s}\left(X+a_{0}\right)^{r}\left(Y+a_{0}\right)^{s}= \\
& =X^{p+1}+Y^{p+1}+\sum_{r=1}^{p} \sum_{s=1}^{p} d_{r s} X^{r} Y^{s} .
\end{aligned}
$$

On expanding the binomials on the left-hand side and comparing the coefficients of the different power products of $X$ and $Y$, we find that the terms in $X^{p+1}$ and $Y^{p+1}$ cancel out, and that the terms in $X^{r} Y^{s}$, where $r$ and $s$ run from 1 to $p$, just allow to express the coefficients $d_{r s}$ of $G(X, Y)$ as unique linear polynomials in the coefficients $c_{r s}$. The only remaining conditions still to be satisfied are that the coefficients of the powers $1, X, X^{2}, \cdots, X^{p}$ are equal to zero; by symmetry, this implies then the same for the coefficients of the powers $1, Y, Y^{2}, \cdots, Y^{p}$.

These $p+1$ conditions can also be obtained by simply putting $Y=0$ in (32). They are then contained in the single condition that

$$
\left(X+a_{0}\right)^{p+1}+a_{0}^{p+1}+\sum_{r=1}^{p} \sum_{s=1}^{p} c_{r s}\left(X+a_{0}\right)^{r} a_{0}^{s}=X^{p+1}
$$

identically in $X$.

If now the prime $p$ is equal to 2 , then on putting $X=-a_{0}$ we obtain the condition that $a_{0}^{3}=\left(-a_{0}\right)^{3}$, hence that $a_{0}=0$, contrary to hypothesis. Thus the following special result holds.

THEOREM 7. There are no $R_{2}$-series of order 0 . In other words, if $f(z)$ is an $S_{2}$-series of order 0 , then its reciprocal $f(z)^{-1}$ is not an $S_{2}$-series.

A completely different position holds if the prime $p$ is greater than 2 .

Now, on replacing $X$ by $X-a_{0}$ in (33), this identity takes the form

$$
X^{p+1}+a_{0}^{p+1}+\sum_{r=1}^{p} \sum_{s=1}^{p} c_{r s} X^{r} a_{0}^{s}=\sum_{r=0}^{p+1}\left(\begin{array}{c}
p+1 \\
r
\end{array}\right) X^{r}\left(-a_{0}\right)^{p-r+1} .
$$

Here the terms in $X^{p+1}$ and in $a_{0}^{p+1}$ cancel out, and the remaining terms lead to the following system of $p$ linear equations

$$
\sum_{s=1}^{p} c_{r s} a_{o}^{s}=\left(\begin{array}{c}
p+1 \\
r
\end{array}\right)\left(-a_{0}\right)^{p-r+1} \quad(r=1,2, \cdots, p)
$$


for the coefficients $c_{r s}$. Each of the $p$ diagonal coefficients $c_{r r}$ occurs in one and only one of these equations and has the coefficient $a_{0}^{\prime} \neq 0$. These $p$ equations are therefore non-contradictory and allow to express the $c_{r}$ linearly in terms of the remaining

$$
Q-p=\frac{p(p-1)}{2}
$$

essential coefficients

$$
c_{r s}, \quad \text { where } 1 \leqq r<s \leqq p,
$$

and of the coefficient $a_{0}$. Thus the following result has been established.

For $p \geqq 3$ the set of all $R_{p}$-series of order 0 depends on $Q-p+1$ parameters in $K$, viz., on the coefficients (35) and on $a_{0}$.

Since both sides of the equation (34) have the common factor $a_{0}$, we further find that if $F(X, Y)$ is given, $a_{0}$ has at most $p-1$ possible values. For otherwise these equations are satisfied identically in $a_{0}$, and hence

$$
c_{r, p-r+1}=(-1)^{p-r+1}\left(\begin{array}{c}
p+1 \\
r
\end{array}\right) \text { for } r=1,2, \cdots, p, \text { and otherwise } c_{r s}=0 .
$$

Therefore

$$
F(X, Y)=X^{p+1}+Y^{p+1}+\sum_{r=1}^{p}(-1)^{p-r+1}\left(\begin{array}{c}
p+1 \\
r
\end{array}\right) X^{r} Y^{p-r+1}=(X-Y)^{p+1} .
$$

However, the only Laurent series $f(z)$ of order 0 satisfying the functional equation

$$
F\left(f\left(z^{p}\right), f(z)\right)=\left(f\left(z^{p}\right)-f(z)\right)^{p+1}=0
$$

is an arbitrary constant and so is not an $S_{p}$-series.

19. In the preceding sections we studied in detail on how many parameters does the general $S_{p}$-series $f(z)$ depend. The results were found to depend on whether $f(z)$ was of negative order, of positive order, or of order 0 .

It was also found that every $S_{p}$-series could be expressed in terms of the normed $S_{p}$-series $g[z]$ of order -1 , and when we were dealing with $S_{p}$-series of negative order, this normed series could be replaced by the corresponding basic series $h[z]$ in which the constant term was equal to zero.

In the next chapter we shall deal in detail with the relation between such a basic series $h[z]$ and its polynomial $H(X, Y)$.

Before studying this question, it is, however, appropriate to add some remarks on the $S_{p}$-series $f(z)$ for which the corresponding polynomial $F(X, Y)$ is reducible. 
20. Let for the moment $f(z)$ be an $S_{p}$-series of arbitrary order, but with the property that its polynomial $F(X, Y)$ is reducible. By $\S 7, F(X, Y)$ allows then a factorisation

$$
F(X, Y)=h H(X, Y) H(Y, X)
$$

where $h \neq 0$ is a constant, and $H(X, Y)$ is an irreducible polynomial which has the exact degree 1 in $X$ and $p$ in $Y$, hence without loss of generality, may be assumed to have the form

$$
H(X, Y)=-X+\sum_{s=0}^{p} \gamma_{s} Y^{s}
$$

where $\gamma_{0}, \cdots, \gamma_{p-1}, \gamma_{p} \neq 0$ are certain constants. Hence

$$
F(X, Y)=h\left(-Y+\sum_{r=0}^{p} \gamma_{r} X^{r}\right)\left(-X+\sum_{s=0}^{p} \gamma_{s} Y^{s}\right) .
$$

Since $X^{p+1}$ is the term of $F(X, Y)$ in the highest power of $S$, it follows at once that

$$
h \gamma_{p}=-1 .
$$

Let now, firstly, $f(z)$ be the negative order $m$, and let it be normed,

$$
f(z)=z^{m}+\cdots \text {. }
$$

The identity

$$
H\left(f\left(z^{p}\right), f(z)\right)=0,
$$

which has already been used before, takes the explicit form

$$
-\left(z^{m p}+\cdots\right)+\sum_{s=0}^{p} \gamma_{s}\left(z^{m}+\cdots\right)^{s}=0
$$

Its term in $z^{m p}$ has the coefficient $-1+\gamma_{p}$ which must vanish; hence

$$
\gamma_{p}=1, \quad h=-1 \text {. }
$$

Hence the following result holds for reducible $F(X, Y)$.

If $f(z)$ is normed and of negative order, then its polynomial has the form

$$
F(X, Y)=-\left(X^{p}-Y+\sum_{r=0}^{p-1} \gamma_{r} X^{r}\right)\left(Y^{p}-X+\sum_{s=0}^{p-1} \gamma_{s} Y^{s}\right),
$$

hence depends on p parameters $\gamma_{0}, \gamma_{1}, \cdots, \gamma_{p-1}$ in $K$.

We must add $a_{m} \neq 0$ as a further parameter if $f(z)$ is not normed. A similar proof shows that if $f(z)$ is basic, then also $\gamma_{p-1}=0$. 
21. The two remaining cases lead to rather trivial results, as follows.

Let, secondly, $f(z)$ be of positive order and normed. Then, on one hand, the reducible polynomial $F(X, Y)$ has the form (36). On the other hand, it follows from $\S 9$ that $F(X, Y)$ has the special form

$$
F(X, Y)=X^{p+1}+Y^{p+1}+\sum_{r=1}^{p} \sum_{s=1}^{p} c_{r s} X^{r} Y^{s},
$$

and here, by $\S \S 11-12$,

$$
c_{p p}=-1 .
$$

On comparing these two expressions for $F(X, Y)$, it follows immediately that

$$
\gamma_{0}=\gamma_{1}=\cdots=\gamma_{p-1}=0, \quad \gamma_{p}=1, \quad h=-1
$$

and that therefore

$$
F(X, Y)=-\left(X^{p}-Y\right)\left(Y^{p}-X\right) .
$$

The corresponding equation

$$
H\left(f\left(z^{p}\right), f(z)\right)=f\left(z^{p}\right)-f(z)^{p}=0
$$

is easily seen to have no other normed solution of the positive order $m$ than the monomial $f(z)=z^{m}$. The general $S_{p}$-series of positive order with a reducible polynomial $F(X, Y)$ has the form $f(z)=a_{m} z^{m}$ where again $a_{m} \neq 0$ is a constant.

Thirdly, let $f(z)$ be of order 0 and not necessarily normed. Since $f(z)-a_{0}$ is of positive order and still has a reducible polynomial, it follows now that $f(z)$ is a polynomial

$$
f(z)=a_{0}+a_{m} z^{m}, \text { where } a_{0} \neq 0 \text { and } a_{m} \neq 0
$$

are arbitrary constants and $m$ is any positive integer. The polynomial $F(X, Y)$ of $f(z)$ has then the special form

$$
F(X, Y)=-\left(\left(X-a_{0}\right)^{p}-\left(Y-a_{0}\right)\right)\left(\left(Y-a_{0}\right)^{p}-\left(X-a_{0}\right)\right) .
$$

In future an $S_{p}$-series $f(z)$ will be called general or special, according as to whether its polynomial $F(X, Y)$ is irreducible or reducible, respectively. By what has just been proved, special $S_{p}$-series of non-negative orders are monomial or binomial polynomials and so have little interest. On the other hand, special $S_{p}$-series of negative orders have non-trivial properties.

\section{Chapter 2. Basic series and their polynomials}

22. As was found in the first chapter, $S_{p}$-series of negative orders are more general than those of non-negative orders, and the latter can always be expressed in terms of $S_{p}$-series of negative orders. 
It is for this reason that we shall from now on consider only $S_{p}$-series of negative orders. It will, in fact, be sufficient to deal only with basic series, i.e., with normed series of order -1 in which the constant term is missing.

Let

$$
h[z]=z^{-1}+\sum_{h=1}^{\infty} b_{h} z^{h}
$$

be such a basic $S_{p}$-series, and let, as in $\S 15$, its polynomial $H(X, Y)$ be written in the form

$$
H(X, Y)=-\left(X^{p}-Y\right)\left(Y^{p}-X\right)+\sum_{r=0}^{p} \sum_{s=0}^{p} E_{r s} X^{r} Y^{s} \quad\left(E_{r s}=E_{s r}\right) .
$$

As was then already mentioned, the coefficients of $H(X, Y)$ satisfy the conditions

$$
E_{p p}=E_{p-1, p}=E_{p, p-1}=0 .
$$

We note once more that every $S_{p}$-series of negative order $m$ can be expressed in the form

$$
f(z)=a_{0}+a_{m} h\left[z^{-m}\right]
$$

where $a_{0}$ and $a_{m} \neq 0$ are arbitrary constants, and that then, by $\S 8$, the polynomial $F(X, Y)$ of $f(z)$ is given by

$$
a_{m}^{p+1} F(X, Y)=H\left(a_{0}+a_{m} X, a_{0}+a_{m} Y\right) .
$$

23. Theorem 4 and its corollary imply that, when $H(X, Y)$ is given, the basic $S_{p}$-series $h[z]$ is unique, and all its coefficients $b_{h}$ can be expressed as polynomials in the coefficients $E_{r s}$ of $H(X, Y)$. It was then already mentioned that, conversely, it is similarly possible to write the coefficients $H_{r s}$ as polynomials in a certain finite number of the coefficients $b_{h}$. This representation will now be established, but explicitly only in the lowest two cases when $p=2$ and $p=3$ because for larger primes $p$ the formulae become rather complicated.

The idea, on which this calculation is based, is, however, quite simple. It is founded on the definition of $H(X, h[z])$ as the monic polynomial in $X$ of degree $p+1$ which has as its zeros the $p+1$ elements

$$
h\left[z^{p}\right], h\left[z^{1 / p}\right], h\left[\varepsilon z^{1 / p}\right], \cdots, h\left[\varepsilon^{p-1} z^{1 / p}\right]
$$

of the set $\Sigma_{h}$. It is thus required to evaluate the $p+1$ elementary symmetric functions, $s_{1}, s_{2}, \cdots, s_{p+1}$ say, of these $p+1$ series, and then

$$
H(X, h[z])=X^{p+1}-s_{1} X^{p}+s_{2} X^{p-1}-+\cdots \pm s_{p+1} .
$$


Here the main problem lies in expressing the $s_{i}$ as polynomials in $h[z]$ and in the Laurent coefficients $b_{h}$.

24. To begin with, the definition (38) of $h[z]$ is easily seen to imply an infinite sequence of identities

$$
\begin{aligned}
& z^{-1}=h[z]+\cdots \\
& z^{-2}=h[z]^{2}-2 b_{1}+\cdots, \\
& z^{-3}=h[z]^{3}-3 b_{1} h[z]-3 b_{2}+\cdots, \\
& z^{-4}=h[z]^{4}-4 b_{1} h[z]^{2}-4 b_{2} h[z]+\left(2 b_{1}^{2}-4 b_{3}\right)+\cdots,
\end{aligned}
$$

etc., when the dots denote formal power series in negative powers of $h[z]$. Further positive powers of $z$ are expressible as formal power series in such negative powers.

Consider now, firstly, the simplest case when $p=2$. The set $\Sigma_{h}$ consists of the three series

$$
\begin{gathered}
h\left[z^{2}\right]=z^{-2}+\sum_{h=1}^{\infty} b_{h} z^{2 h} \\
h\left[z^{1 / 2}\right]=z^{1 / 2}+\sum_{h=1}^{\infty} b_{h} z^{h / 2}, \quad h\left[-z^{1 / 2}\right]=-z^{-1 / 2}+\sum_{h=1}^{\infty}(-1)^{h} z^{h / 2} .
\end{gathered}
$$

Here

$$
h\left[z^{1 / 2}\right]+h\left[-z^{1 / 2}\right]=2 \sum_{h=1}^{\infty} b_{2 h} z^{h} \quad \text { and } \quad h\left[z^{1 / 2}\right] h\left[-z^{1 / 2}\right]=-z^{-1}-\sum_{h=0}^{\infty} \beta_{h} z^{h},
$$

where the new coefficients $\beta_{h}$ are defined by

$$
\begin{gathered}
\beta_{0}=2 b_{1}, \quad \beta_{1}=2 b_{3}+b_{1}^{2}, \quad \text { and } \\
\beta_{h}=2 b_{2 h+1}+2 \sum_{j=1}^{h-1}(-1)^{j-1} b_{j} b_{2 h-j}+(-1)^{h-1} b_{h}^{2} \text { for } h \geqq 2 .
\end{gathered}
$$

The three elementary symmetric functions

$$
\begin{aligned}
& s_{1}=h\left[z^{2}\right]+h\left[z^{1 / 2}\right]+h\left[-z^{1 / 2}\right], \\
& s_{2}=h\left[z^{2}\right] h\left[z^{1 / 2}\right]+h\left[z^{2}\right] h\left[-z^{1 / 2}\right]+h\left[z^{1 / 2}\right] h\left[-z^{1 / 2}\right], \\
& s_{3}=h\left[z^{2}\right] h\left[z^{1 / 2}\right] h\left[-z^{1 / 2}\right]
\end{aligned}
$$

can therefore be written as the Laurent series 


$$
\begin{aligned}
& s_{1}=z^{-2}-2 b_{1}+\cdots, \\
& s_{2}=\left(2 b_{2}-1\right) z^{-1}+\left(2 b_{4}-2 b_{1}\right)+\cdots, \\
& s_{3}=-z^{-3}-2 b_{1} z^{-2}-\left(2 b_{3}+b_{1}^{2}\right) z^{-1}-\left(2 b_{5}+2 b_{1} b_{3}-b_{2}^{2}\right)+\cdots,
\end{aligned}
$$

where the dots now denote power series in positive powers of $z$.

Replace now in (42) the terms in non-positive powers of $z$ by their expressions (41); they become then Laurent series in $h[z]$. But we know that $s_{1}, s_{2}$, and $s_{3}$ are polynomials in $h[z]$; hence the terms in negative powers of $h[z]$ must cancel out. The explicit expressions of $s_{1}, s_{2}$, and $s_{3}$ take therefore the form $s_{1}=h[z]^{2}-2 b_{1}$, $s_{2}=\left(2 b_{2}-1\right) h[z]+\left(2 b_{4}-2 b_{1}\right)$, $s_{3}=-h[z]^{3}-2 b_{1} h[z]^{2}-\left(2 b_{3}+b_{1}^{2}-3 b_{1}\right) h[z]-\left(2 b_{5}+2 b_{1} b_{3}-b_{2}^{2}-3 b_{2}-4 b_{1}^{2}\right)$.

From these expressions, it follows immediately that the polynomial $H(X, Y)$ of $h[z]$ has the following explicit form,

$$
\begin{aligned}
H(X, Y)= & -\left(X^{2}-Y\right)\left(Y^{2}-X\right)+2 b_{1}\left(X^{2}+Y^{2}\right)+2 b_{2} X Y+\left(2 b_{4}-2 b_{1}\right) X+ \\
& +\left(2 b_{3}+b_{1}^{2}-3 b_{1}\right) Y+\left(2 b_{5}+2 b_{1} b_{3}-b_{2}^{2}-3 b_{2}-4 b_{1}^{2}\right) .
\end{aligned}
$$

By Theorem 2, $H(X, Y)$ is symmetric in $X$ and $Y$. Hence

$$
2 b_{4}=2 b_{3}+b_{1}^{2}-b_{1} \text {. }
$$

The essential coefficients $H_{r s}$ of $H(X, Y)$ are given by

$$
\begin{gathered}
H_{02}=2 b_{1}, \quad H_{11}=2 b_{2}, \\
H_{01}=2 b_{3}+b_{1}^{2}-3 b_{1}=2 b_{4}-2 b_{1}, \quad H_{00}=2 b_{\varsigma}+2 b_{1} b_{3}-b_{2}^{2}-3 b_{2}-4 b_{1}^{2},
\end{gathered}
$$

and, conversely,

$$
\begin{gathered}
2 b_{1}=H_{02}, \quad 2 b_{2}=H_{11}, \\
8 b_{3}=4 H_{01}-H_{02}^{2}+6 H_{02}, \quad 2 b_{4}=H_{01}+H_{02}, \\
16 b_{5}=8 H_{00}-4 H_{02} H_{01}+H_{02}^{3}+2 H_{02}^{2}-2 H_{11}^{2}+6 H_{11} .
\end{gathered}
$$

Thus for basic $S_{2}$-series $h[z]$ the polynomial $H(X, Y)$ is known if the first five coefficients

$$
b_{1}, b_{2}, b_{3}, b_{4}, b_{5}
$$

are given where, however, these five coefficients have to satisfy the condition (44). Theorem 4 shows that no further restrictions on the coefficients (45) need be imposed. Thus, when the equation (44) holds, then there always exists exactly one basic $S_{2}$-series with the first five coefficients (45). 
It should now be possible to express all further coefficients $b_{h}(h \geqq 6)$ of $h[z]$ in terms of the coefficients (45). As we shall in fact immediately prove, these coefficients $b_{h}$ with $h \geqq 6$ can be written as polynomials in the coefficients (45), where the numerical coefficients lie in the prime field of $K$.

25. For this purpose, a set of recursive formulae will now be established which allows to evaluate step by step the successive coefficients $b_{h}$ once the coefficients (45) are given. Thus these recursive formulae depend only implicitly on the polynomial $H(X, Y)$ of $h[z]$.

The construction is based on the two identities

$$
\begin{gathered}
s_{1}=h\left[z^{2}\right]+h\left[z^{1 / 2}\right]+h\left[-z^{1 / 2}\right]=h[z]^{2}-2 b_{1}, \\
s_{2}=h\left[z^{2}\right]\left(h\left[z^{1 / 2}\right]+h\left(-z^{1 / 2}\right]\right)+h\left[z^{1 / 2}\right] h\left[-z^{1 / 2}\right]=\left(2 b_{1}-1\right) h[z]+\left(2 b_{4}-2 b_{1}\right)
\end{gathered}
$$

of the last section. If in these identities

$$
h[z], h\left[z^{2}\right], h\left[z^{1 / 2}\right], h\left[-z^{1 / 2}\right]
$$

are replaced by their Laurent series, the resulting identities contain only integral powers of $z$. On comparing now the coefficients of these different powers and putting their sums equal to 0 , the required recursive formulae are obtained. By means of a suitable linear combination, they can then be put in the following forms where $k$ runs over all positive integers, and empty sums are to mean 0 .

$$
\begin{gathered}
b_{4 k}=b_{2 k+1}+\sum_{j=1}^{k-1} b_{j} b_{2 k-j}+\left(b_{k}^{2}-b_{k}\right) / 2 \\
b_{4 k+1}=b_{2 k+3}+\sum_{j=1}^{k} b_{j} b_{2 k-j+2}-\sum_{j=1}^{2 k-1}(-1)^{j-1} b_{j} b_{4 k-j}+ \\
+\sum_{j=1}^{k-1} b_{j} b_{4 k-4 j}-b_{2} b_{2 k}+\left(b_{k+1}^{2}-b_{k+1}\right) / 2+\left(b_{2 k}^{2}+b_{2 k}\right) / 2 \\
b_{4 k+2}=b_{2 k+2}+\sum_{j=1}^{k} b_{i} b_{2 k-j+1}, \\
b_{4 k+3}=b_{2 k+4}+\sum_{j=1}^{k+1} b_{j} b_{2 k-j+3}-\sum_{j=1}^{2 k}(-1)^{j-1} b_{j} b_{4 k-j+2}+ \\
+\sum_{j=1}^{k} b_{j} b_{4 k-4 j+2}-b_{2} b_{2 k+1}-\left(b_{2 k+1}^{2}-b_{2 k+1}\right) / 2 .
\end{gathered}
$$

There is a third identity

$$
\begin{aligned}
s_{3} & =h\left[z^{2}\right] h\left[z^{1 / 2}\right] h\left[-z^{1 / 2}\right]= \\
& =-\left(h[z]^{3}+2 b_{1} h[z]^{2}+\left(2 b_{3}+b_{1}^{2}-3 b_{1}\right) h[z]+\left(2 b_{5}+2 b_{1} b_{3}-b_{2}^{2}-3 b_{2}-4 b_{1}^{2}\right)\right),
\end{aligned}
$$


to which the same method may be applied. This leads then to a further set of recursive formulae. But while the formulae (46) involve at most products of two coefficients $b_{h}$, and in addition have rather simple laws, the new formulae contain products of up to three factors $b_{h}$ and are much more complicated. The first three of the new relations are as follows.

$$
\begin{aligned}
2 b_{7} & +2 b_{1} b_{5}-2 b_{2} b_{4}+b_{3}^{2}= \\
= & b_{1}\left(3 b_{3}+3 b_{1}^{2}\right)+4 b_{1} b_{2}+\left(2 b_{3}+b_{1}^{2}-3 b_{1}\right) b_{1}, \\
2 b_{9} & +2 b_{1} b_{7}-2 b_{2} b_{6}+2 b_{3} b_{5}-b_{4}^{2}= \\
= & 2 b_{1}^{2}+\left(4 b_{4}+4 b_{1} b_{2}\right)+2 b_{1}\left(2 b_{3}+b_{1}^{2}\right)+\left(2 b_{3}+b_{1}^{2}-3 b_{1}\right) b_{2}, \\
2 b_{11} & +2 b_{1} b_{9}-2 b_{2} b_{8}+2 b_{3} b_{7}-2 b_{4} b_{6}+b_{5}^{2}= \\
= & b_{1}\left(2 b_{3}+b_{1}^{2}\right)+b_{2}+\left(3 b_{5}+6 b_{1} b_{3}+3 b_{2}^{2}+b_{1}^{3}\right)+ \\
& +2 b_{1}\left(2 b_{4}+2 b_{1} b_{2}\right)+\left(2 b_{3}+b_{1}^{2}-3 b_{1}\right) b_{3},
\end{aligned}
$$

and the further ones get even more involved. We can fortunately disregard all these formulae because, by Theorem 4, they are necessarily consequences of (44) and of the recursive formulae (46).

26. Next let $p=3$, and let

$$
h[z]=z^{-1}+\sum_{h=1}^{\infty} b_{h} z^{h}
$$

be any basic $S_{3}$-series. The corresponding set $\Sigma_{h}$ consists of the four series

$$
h\left[z^{3}\right]=z^{-3}+\sum_{h=1}^{\infty} b_{h} z^{3 h}
$$

and $h\left[\varepsilon^{i} z^{1 / 3}\right]=\varepsilon^{-j} z^{-1 / 3}+\sum_{h=1}^{\infty} b_{h} \varepsilon^{h i} z^{h / 3}$ where $j=0,1,2$.

Here $\varepsilon$ denotes a primitive third root of unity in $K$.

The problem is again to express the elementary symmetric functions $s_{1}, s_{2}$, $s_{3}, s_{4}$ of the elements of $\Sigma_{h}$ as polynomials in $h[z]$. This can be done just as for $p=2$, but the calculations become now more complicated. On substituting the expressions so obtained for the $s_{i}$ in $H(X, Y)$, this polynomial assumes the following explicit form: 


$$
\begin{aligned}
H(X, Y) & = \\
= & -\left(X^{3}-Y\right)\left(Y^{3}-X\right)+3 b_{1}\left(X^{3} Y+X Y^{3}\right)+3 b_{2}\left(X^{3}+Y^{3}\right)+3 b_{3} X^{2} Y^{2}+ \\
& +\left(3 b_{4}+3 b_{1} b_{2}\right) X^{2} Y+3 b_{6} X Y^{2}+\left(3 b_{5}-3 b_{1} b_{3}+3 b_{2}^{2}+b_{1}^{3}-4 b_{1}\right) X^{2}+ \\
& +\left(3 b_{9}-6 b_{1} b_{3}-3 b_{1}\right) Y^{2}+\left(3 b_{7}+3 b_{1} b_{5}+3 b_{2} b_{4}-3 b_{3}^{2}-9 b_{1}^{2}\right) X Y+ \\
(47) \quad & +\left(3 b_{8}-3 b_{1} b_{6}+6 b_{2} b_{5}-3 b_{3} b_{4}+3 b_{1}^{2} b_{4}-3 b_{1} b_{2} b_{3}+b_{2}^{3}-9 b_{1} b_{2}-4 b_{2}\right) X+ \\
& +\left(3 b_{10}+3 b_{1} b_{8}+3 b_{2} b_{7}-6 b_{3} b_{6}+3 b_{4} b_{5}-6 b_{1} b_{4}-6 b_{1}^{2} b_{2}-9 b_{1} b_{2}-3 b_{2}\right) Y+ \\
& +\left(3 b_{11}-3 b_{1} b_{9}+6 b_{2} b_{8}-3 b_{3} b_{7}+3 b_{1}^{2} b_{7}-3 b_{4} b_{6}-3 b_{1} b_{2} b_{6}+3 b_{5}^{2}+\right. \\
& +3 b_{2}^{2} b_{5}-6 b_{1} b_{5}-3 b_{1} b_{3} b_{5}+3 b_{1} b_{4}^{2}-3 b_{2} b_{3} b_{4}+b_{3}^{3}+6 b_{1}^{2} b_{3}- \\
& \left.-4 b_{3}-6 b_{1} b_{2}^{2}-9 b_{2}^{2}-2 b_{1}^{4}+2 b_{1}^{2}+b_{1}\right) .
\end{aligned}
$$

Since $H(X, Y)$ is symmetric in $X$ and $Y$, the Laurent coefficients $b_{h}$ that occur in this representation must satisfy the following three symmetry conditions:

$$
\begin{aligned}
b_{6}= & b_{4}+b_{1} b_{2} \\
b_{9}= & b_{5}+b_{1} b_{3}+b_{2}^{2}+\left(b_{1}^{3}-b_{1}\right) / 3 \\
b_{10}= & -b_{1} b_{8}+b_{8}-b_{2} b_{7}+2 b_{3} b_{6}-b_{1} b_{6}-b_{4} b_{5}+2 b_{2} b_{5}-b_{3} b_{4}+ \\
& +b_{1}^{2} b_{4}+2 b_{1} b_{4}-b_{1} b_{2} b_{3}+2 b_{1}^{2} b_{2}+\left(b_{2}^{3}-b_{2}\right) / 3
\end{aligned}
$$

Thus for $p=3$ the eight Laurent coefficients

$$
b_{1}, b_{2}, b_{3}, b_{4}, b_{5}, b_{7}, b_{8}, b_{11}
$$

of $h[z]$ determine $b_{6}, b_{9}$, and $b_{10}$ and hence also determine the polynomial $H(X, Y)$.

The formulae (47) and (48) allow to write the eight essential coefficients

$$
E_{13}, E_{03}, E_{22}, E_{12}, E_{02}, E_{11}, E_{01}, E_{00}
$$

of $H(X, Y)$ as polynomials in the eight Laurent coefficients (49) of $h[z]$, and conversely, the latter can be expressed as polynomials in the coefficients $(50)$ of $H(X, Y)$.

27. Just as for $p=2$, so also in the present case $p=3$ we can establish recursive formulae for the coefficients $b_{h}$ of $h[z]$. It is to be expected that these formulae will be rather more complicated. They again involve the coefficients of $H(X, Y)$ only implicitly. 
Put

$$
\begin{gathered}
k(z)=\sum_{h=1}^{\infty} b_{h} z^{3 h} ; \\
k_{0}(z)=\sum_{h=1}^{\infty} b_{3 h} z^{h}, \quad k_{1}(z)=\sum_{h=0}^{\infty} b_{3 h+1} z^{h}, \quad k_{2}(z)=\sum_{h=0}^{\infty} b_{3 h+2} z^{h} .
\end{gathered}
$$

The four elements of $\Sigma_{h}$ can then be written in the form

$$
\begin{gathered}
h\left[z^{3}\right]=z^{-3}+k(z) \\
h\left[\varepsilon^{j} z^{1 / 3}\right]=\varepsilon^{-j} z^{-1 / 3}+k_{0}(z)+\varepsilon^{i} z^{1 / 3} k_{1}(z)+\varepsilon^{2 j} z^{2 / 3} k_{2}(z) \text { for } j=0,1,2 .
\end{gathered}
$$

On evaluating their elementary symmetric functions, the terms in fractional powers of $z$ cancel out, and the $s_{j}$ become polynomials in $z, k(z), k_{0}(z), k_{1}(z)$, and $k_{2}(z)$. On the other hand, the expression (47) for $H(X, Y)$ allows to write them also as polynomials in $h[z]$ and in the first eleven coefficients $b_{h}$ of $h[z]$.

On carrying out these trivial calculations, we obtain the following set of formulae.

$$
\begin{aligned}
s_{1}= & h[z]^{3}-3 b_{1} h[z]-3 b_{2}=z^{-3}+k(z)-3 k_{0}(z), \\
s_{2}= & 3 b_{3} h[z]^{2}+3 b_{6} h[z]+\left(3 b_{9}-6 b_{1} b_{3}-3 b_{1}\right)= \\
= & 3 z^{-3} k_{0}(z)+3 k(z) k_{0}(z)+3 k_{0}(z)^{2}-3 k_{1}(z)-3 z k_{1}(z) k_{2}(z) \\
s_{3}= & -3 b_{1} h[z]^{3}-3 b_{6} h[z]^{2}+\left(1-3 b_{7}-3 b_{1} b_{5}-3 b_{2} b_{4}+3 b_{3}^{2}+9 b_{1}^{2}\right) h[z]- \\
& -\left(3 b_{8}-3 b_{1} b_{6}+6 b_{2} b_{5}-3 b_{3} b_{4}+3 b_{1}^{2} b_{4}-3 b_{1} b_{2} b_{3}+b_{2}^{3}-9 b_{1} b_{2}-4 b_{2}\right)= \\
= & 3 z^{-3}\left(k_{0}(z)^{2}-k_{1}(z)-z k_{1}(z) k_{2}(z)\right)+z^{-1}+k_{0}(z)^{3}+z k_{1}(z)^{3}+ \\
& +3 k_{2}(z)+3 z k_{2}(z)^{2}+z^{2} k_{2}(z)^{3}-3 k_{0}(z) k_{1}(z)-3 z k_{0}(z) k_{1}(z) k_{2}(z)+ \\
& +3 k(z)\left(k_{0}(z)^{2}-k_{1}(z)-z k_{1}(z) k_{2}(z)\right) .
\end{aligned}
$$

Here replace again $h[z], k(z), k_{0}(z), k_{1}(z)$, and $k_{2}(z)$, by their series and then compare on both sides of the resulting identities the coefficients of the different powers of $z$ : For the lower powers of $z$ this leads only to trivial identities, or to the symmetry conditions (48), or to equations that follow from these conditions.

But for $h \geqq 4$ the wanted recursive formulae are obtained. They have the following forms:

$$
b_{3 h}=b_{h+2}-b_{1} b_{h}-\frac{1}{3} b_{h / 3}+\sum_{j=1}^{h} b_{j} b_{h-j+1}+\frac{1}{3} \sum_{h_{1}+h_{2}+h_{3}=h} b_{h_{1}} b_{h_{2}} b_{h_{3}},
$$

where $b_{h / 3}$ denotes 0 if $h$ is not divisible by 3 , and in the multiple sum $h_{1}, h_{2}, h_{3}$ denote positive integers of the sum $h$; 


$$
\begin{aligned}
b_{3 h+1}= & -b_{6} b_{h}-2 b_{3} b_{h+1}-b_{1} b_{h+1}-\frac{1}{3} b_{(h+3) / 3}+b_{h+5}-b_{3} \sum_{j=1}^{h-1} b_{j} b_{h-j}+ \\
& +\sum_{i=1}^{h+3} b_{j} b_{h-j+4}+\sum_{j=1}^{h-1} b_{3 j} b_{3 h-3 j}-\sum_{j=0}^{h-1} b_{3 j+1} b_{3 h-3 i-1}+ \\
& +\sum_{1 \leq j<h / 3} b_{j} b_{3 h-9 j}+\frac{1}{3} \sum_{h_{1}+h_{2}+h_{3}=h+3} b_{h_{1}} b_{h_{2}} b_{h_{3}},
\end{aligned}
$$

where again $b_{(h+3) / 3}$ denotes 0 if $h$ is not divisible by 3 , and where in the last sum $h_{1}, h_{2}, h_{3}$ run over all positive integers of the sum $h+3$;

$$
\begin{aligned}
b_{3 h+2}= & \frac{1}{3}\left(1-3 b_{7}-3 b_{1} b_{5}-3 b_{2} b_{4}+3 b_{3}^{2}+9 b_{1}^{2}\right) b_{h}-2 b_{6} b_{h+2}-3 b_{1} b_{h+2}- \\
& -b_{6} b_{h+3}-2 b_{3} b_{h+4}-b_{(h+6) / 3}-b_{1} b_{h+6}+b_{h+8}-b_{6} \sum_{j=1}^{h-1} b_{j} b_{h-j}- \\
& -3 b_{1} \sum_{j=1}^{h} b_{j} b_{h-j+1}-b_{3} \sum_{j=1}^{h+2} b_{j} b_{h-j+3}+\sum_{j=1}^{h+6} b_{j} b_{h-j+7}+ \\
& +\sum_{1 \leq j \leq h / 3} b_{j} b_{3 h-9 j+1}+\sum_{1 \leqq j<(h+3) / 3} b_{j} b_{3 h-9 j+9}+\sum_{j=1}^{h} b_{3 j} b_{3 h-3 j+1}- \\
& -\sum_{j=0}^{h-1} b_{3 j+2} b_{3 h-3 j-1}-b_{1} \sum_{h_{1}+h_{2}+h_{3}=h} b_{h_{1}} b_{h_{2}} b_{h_{3}}+ \\
& +\frac{1}{3} \sum_{h_{1}+h_{2}+h_{3}=h+6} b_{h_{1}} b_{h_{2}} b_{h_{3}}-\sum_{3 h_{1}+h_{2}+h_{3}-h} b_{h_{1}} b_{3 h_{2}} b_{3 h_{3}}+ \\
& +\sum_{3 h_{1}+k_{2}+k_{3}=h-1} b_{h_{1}} b_{3 k_{2}+1} b_{3 k_{3}+2}-\frac{1}{3} \sum_{h_{1}+h_{2}+h_{3}=h} b_{3 h_{1}} b_{3 h_{2}} b_{3 h_{3}}+ \\
& +\sum_{h_{1}+k_{2}+k_{3}=h-1} b_{3 h_{1}} b_{3 k_{2}+1} b_{3 k_{3}+2}-\frac{1}{3} \sum_{k_{1}+k_{2}+k_{3}=h-1} b_{3 k_{1}+1} b_{3 k_{2}+1} b_{3 k_{3}+1}- \\
& -\frac{1}{3} \sum_{k_{1}+k_{2}+k_{3}=h-2} b_{3 k_{1}+2} b_{3 k_{2}+2} b_{3 k_{3}+2},
\end{aligned}
$$

where also $b_{(h+6) / 3}$ denotes 0 if $h$ is not divisible by 3 , and where in the multiple sums letters $h_{i}$ run over positive integers and letters $k_{i}$ over non-negative integers.

There is a further set of recursive formulae corresponding to the two expressions for the symmetric function $s_{4}$, but I have not tried to determine this set since it is naturally a consequence of the three sets (51).

28. For $p \geqq 5$ I have not tried to determine $H(X, Y)$ and the full sets of 
recursive formulae analogous to (46) for $p=2$ and (51) for $p=3$; for they become very complicated.

We note, however, that the first symmetric function

$$
s_{1}=h\left[z^{p}\right]+\sum_{j=0}^{p-1} h\left[\varepsilon^{j} z^{1 / p}\right],
$$

where $\varepsilon$ denotes again a primitive $p$ th root of unity in $K$, may still easily be written as a polynomial in $h[z]$, so that at least for the special coefficients $b_{h p}$ a set of recursive formulae can be deduced. This is done as follows.

In the formula (41) of $\$ 24$, we had for $1 \leqq n \leqq 4$ written the expressions for the terms in non-negative powers of $h[z]$ of the developments of $z^{-n}$ into Laurent series in descending powers of $h[z]$. For $n=5$ and $n=7$ one similarly finds that

$$
z^{-5}=h[z]^{5}-5 b_{1} h[z]^{3}-5 b_{2} h[z]^{2}+5\left(b_{1}^{2}-b_{3}\right) h[z]+5\left(b_{1} b_{2}-b_{4}\right)+\cdots
$$

and

$$
z^{-7}=h[z]^{7}-7 b_{1} h[z]^{5}-7 b_{2} h[z]^{4}+7\left(2 b_{1}^{2}-b_{3}\right) h[z]^{3}+7\left(3 b_{1} b_{2}-b_{4}\right) h[z]^{2}+
$$

$$
+7\left(2 b_{1} b_{3}+b_{2}^{2}-b_{2}^{3}-b_{5}\right) h[z]+7\left(b_{1} b_{4}+b_{2} b_{3}-b_{1}^{2} b_{2}-b_{6}\right)+\cdots
$$

From the definition of $s_{1}$ as the first elementary symmetric function of the elements of $\Sigma_{h}$ it follows, on the other hand, that

$$
s_{1}=z^{-p}+\sum_{h=1}^{\infty} b_{h} z^{h p}+p \sum_{h=1}^{\infty} b_{h p} z^{h}
$$

Replace now again $h[z]$ in (52) and (53) by its Laurent series and compare the coefficients of the different powers $z^{h}$, where $h \geqq 1$, in the resulting series with those in the series (54). We obtain then the required recursive formulae for $b_{h p}$ in the two cases when $p=5$ or $p=7$.

We shorten these formulae by adopting the following notations. Similarly as before, put $b_{h / p}$ equal to 0 if $h$ is not divisible by $p$. Further, for any two positive integers $m$ and $n$ put

$$
\sigma(m ; n)=\sum b_{h_{1}} b_{h_{2}} \cdots b_{h_{m}}
$$

where the summation is extended over all sets of $m$ positive indices $h_{1}, h_{2}, \cdots, h_{m}$ which satisfy the equation

$$
h_{1}+h_{2}+\cdots+h_{m}=n,
$$

but put the sum equal to 0 if it is empty.

For $p=5$ the resulting formula is still reasonably simple, viz. 


$$
\begin{aligned}
b_{5 h}= & b_{h+4}-3 b_{1} b_{h+2}-2 b_{2} b_{h+1}+\left(b_{1}^{2}-b_{3}\right) b_{h}+2 \sigma(2 ; h+3)- \\
& -3 b_{1} \sigma(2 ; h+1)-b_{2} \sigma(2 ; h)+2 \sigma(3 ; h+2)-b_{1} \sigma(3 ; h)+ \\
& +\sigma(4 ; h+1)+\frac{1}{5}\left(\sigma(5 ; h)-b_{h / 5}\right)
\end{aligned}
$$

It becomes the trivial identity for $h=1$, but for $2 \leqq h \leqq 5$ is equivalent to

$$
\begin{gathered}
b_{10}=b_{6}+b_{1} b_{4}+b_{2} b_{3} \\
b_{15}=b_{7}+b_{1} b_{5}+2 b_{2} b_{4}+b_{3}^{2}+b_{1}^{2} b_{3}+b_{1} b_{2}^{2} \\
b_{20}=b_{8}+b_{1} b_{6}+2 b_{2} b_{5}+3 b_{3} b_{4}+b_{2}^{3}+4 b_{1} b_{2} b_{3}+b_{1}^{2} b_{4}+b_{1}^{3} b_{3} \\
b_{25}=b_{9}+b_{1} b_{7}+2 b_{2} b_{6}+b_{1}^{2} b_{5}+3 b_{3} b_{5}+2 b_{4}^{2}-2 b_{1} b_{2} b_{4}+3 b_{2} b_{3}+b_{1}^{3} b_{3}+ \\
+3 b_{1}^{2} b_{2}^{2}+\frac{1}{5}\left(b_{1}^{5}-b_{1}\right)-b_{2}^{3}
\end{gathered}
$$

Here the equations for $b_{10}$ and $b_{15}$ occurred already amongst the recursive formulae for $p=2$, and for $p=3$, respectively.

For the prime $p=7$ the expression for $b_{7 n}$ is far more complicated.

$$
\begin{aligned}
b_{7 h}= & b_{h+6}-5 b_{1} b_{h+4}-4 b_{2} b_{h+3}+3\left(2 b_{1}^{2}-b_{3}\right) b_{h+2}+2\left(3 b_{1} b_{2}-b_{4}\right) b_{h+1}+ \\
& \left(2 b_{1} b_{3}+b_{2}^{2}-b_{1}^{3}-b_{5}\right) b_{h}+3 \sigma(2 ; h+5)-10 b_{1} \sigma(2 ; h+3)- \\
& -6 b_{2} \sigma(2 ; h+2)+3\left(2 b_{1}^{2}-b_{3}\right) \sigma(2 ; h+1)+ \\
& +\left(3 b_{1} b_{2}-b_{4}\right) \sigma(2 ; h)+5 \sigma(3 ; h+4)-10 b_{1} \sigma(3 ; h+2)- \\
& -4 b_{2} \sigma(3 ; h+1)+\left(2 b_{1}^{2}-b_{3}\right) \sigma(3 ; h)+5 \sigma(4 ; h+3)- \\
& -5 b_{1} \sigma(4 ; h+1)-b_{2} \sigma(4 ; h)+3 \sigma(5 h+2)-b_{1} \sigma(5 ; h)+ \\
& +\sigma(6 ; h+1)+\frac{1}{7}\left(\sigma(7 ; h)-b_{h / 7}\right) .
\end{aligned}
$$

For $h=1$ this formula becomes again the trivial identity, but for $h=2$ and $h=3$ it becomes

$$
\begin{gathered}
b_{14}=b_{8}+b_{1} b_{6}+b_{2} b_{5}+b_{3} b_{4} \\
b_{21}=b_{9}+b_{1} b_{7}+2 b_{2} b_{6}+2 b_{3} b_{5}+b_{1}^{2} b_{5}+b_{4}^{2}+2 b_{1} b_{2} b_{4}+b_{1} b_{3}^{2}+b_{2}^{2} b_{3} .
\end{gathered}
$$

These two equations occurred already amongst the recursive formulae for $p=2$, and for $p=3$, respectively.

29. As we mentioned already, the explicit determination of $H(X, Y)$ for $p \geqq 5$ in terms of the Laurent coefficients $b_{h}$ of $h[z]$ becomes excessively 
complicated. But it is still possible to formulate some general laws about this interdependence.

By the corollary to Theorem 4, each of the Laurent coefficients $b_{h}$ can be expressed as a polynomial over $K$ in the coefficients $E_{r s}$ of $H(X, Y)$. Conversely, the formulae (43) and (44), and (47) and (48), show that for $p=2$ the $E_{r s}$ are polynomials in the four Laurent coefficients

$$
b_{1}, b_{2}, b_{3}, \quad \text { and } b_{5},
$$

and for $p=3$ in the eight Laurent coefficients

$$
b_{1}, b_{2}, b_{3}, b_{4}, b_{5}, b_{7}, b_{8} \text {, and } b_{11} \text {. }
$$

In each of these two cases a multiple of the highest coefficient $\left(2 b_{5}\right.$ or $\left.3 b_{11}\right)$ occurs in the constant term $E_{00}$ of $H(X, Y)$.

An analogous result is still valid for $p \geqq 5$. Also here the highest occurring Laurent coefficient, $b_{h}$. say, arises in the constant term $E_{(0)}$ of $H(X, Y)$, and its suffix $h^{*}$ can be found by the following simple consideration.

The highest elementary symmetric function $s_{p+1}$ of the elements of $\Sigma_{h}$ is the product

$$
s_{p+1}=\left(z^{-p}+\sum_{h=1}^{\infty} b_{h} z^{h p}\right) \prod_{j=0}^{p-1}\left(\varepsilon^{-i} z^{-1 / p}+\sum_{h=1}^{\infty} b_{h} \varepsilon^{i h} z^{h / p}\right) .
$$

Since $p$ is odd, it can also be written as

$$
s_{p+1}=h[z]^{p+1}+\sum_{s=0}^{p} E_{0 s} h[z]^{s} .
$$

On replacing in the second formula $h[z]$ by its Laurent series, two different developments of $s_{p+1}$ into Laurent series are obtained, and on comparing them, it is obvious that the coefficient of $z^{0}$ will form a part of $E_{00}$ and at the same time will involve the Laurent coefficient $b_{h}$. The product formula for $s_{p+1}$ shows easily that the coefficient of $z^{0}$ involves a term

$$
p b_{p^{2}+p-1}
$$

but none with a factor $b_{h}$ where $h \geqq p^{2}+p$. Hence $h^{*}=p^{2}+p-1$. It is also clear that $b_{h}$. cannot occur in any one of the other elementary symmetric functions $s_{1}, s_{2}, \cdots, s_{p}$.

Thus $H(X, Y)$ is already determined if all the Laurent coefficients

$$
b_{h}, \text { where } 1 \leqq h \leqq p^{2}+p-1,
$$

are given.

On the other hand, $H(X, Y)$ depends by 


$$
E_{p p}=E_{p-1, p}=E_{p, p-1}=0
$$

on exactly

$$
P-2=\frac{p(p+3)}{2}-1
$$

essential coefficients $E_{r s}$, and the $p^{2}+p-1$ Laurent coefficients (57) can be expressed as polynomials in the latter.

This implies that the coefficients (57) have still to satisfy

$$
\left(p^{2}+p-1\right)-\left(\frac{p(p+3)}{2}-1\right)=\frac{p(p-1)}{.2}
$$

independent algebraic conditions. Denote by $C_{p}$ this set of $p(p-1) / 2$ conditions.

For $p=2$,

$$
p^{2}+p-1=5, \quad \frac{p(p-1)}{2}=1,
$$

and for $p=3$,

$$
p^{2}+p-1=11, \quad \frac{p(p-1)}{2}=3,
$$

in agreement with the results in $\$ 24$ and $\$ 26$.

The proof just given does not supply any information about the form of the equations in $C_{p}$. Presumably these equations will again be equivalent to the symmetry conditions

$$
E_{r s}=E_{s r} \quad(r, s=0,1, \cdots, p) .
$$

It seems further plausible that in each of the equations of $C_{p}$ the coefficient $b_{h}$ of largest suffix will occur linearly, just as it was for $p=2$ and $p=3$.

The Laurent coefficients

$$
b_{h}, \quad \text { where } \quad h \geqq p^{2}+p,
$$

do not occur in the explicit expression for $H(X, Y)$, but, by the corollary to Theorem 4, they can be expressed as polynomials in the coefficients $E_{r s}$ of $H(X, Y)$. The latter, on the other hand, can be written as polynomials in the $p^{2}+p-1$ Laurent coefficients (57). Hence the coefficients (59) can all be expressed as polynomials in the coefficients (57). This suggests that they are already polynomials in only certain

$$
\frac{p(p+3)}{2}-1
$$


of the coefficients (57). This assertion certainly holds in the two lowest cases $p=2$ and $p=3$, as is obvious from the form of the equations (44), and (48), respectively.

\section{Chapter 3. Analytic $S_{p}$-functions}

30. So far, only formal $S_{p}$-series over a field $K$ have been considered. Here the only restrictions on $K$ were that its characteristic was distinct from $p$ and that it contained a primitive $p$ th root of unity.

Now specialise $K$ and assume that it possesses a non-trivial valuation $w(x)$. The question arises then whether a given $S_{p}$-series over $K$ has a region of convergence relative to the metric induced by the valuation if the indeterminate $z$ is replaced by a variable in $K$.

We shall restrict the discussion of this problem to the particularly interesting case when $K$ is the complex number field $C$, and $w(x)$ is the absolute value $|x|$ in $\underline{C}$.

A given $S_{p}$-series

$$
f(z)=\sum_{n=m}^{\infty} a_{h} z^{n}
$$

with coefficients in $C$ may now possibly converge for

$$
0<|z|<\gamma
$$

if $\gamma$ is a sufficiently small positive number. If this is the case, then $f(z)$ becomes a single-valued analytic function for $|z|<\gamma$ with at most a pole at $z=0$.

It will be sufficient to study this convergence problem for basic $S_{p}$-series

$$
h[z]=z^{-1}+\sum_{h=1}^{\infty} b_{h} z^{h}
$$

For by the results of the first chapter every $S_{p}$-series can be expressed in a trivial way in terms of a suitable basic series.

31. Let then $h[z]$ be any basic $S_{p}$-series with complex coefficients, and let further

$$
H(X, Y)=-\left(X^{P}-Y\right)\left(Y^{P}-X\right)+\sum_{r=0}^{p} \sum_{s=0}^{p} E_{r s} X^{r} Y^{s} \quad\left(E_{r s}=E_{s r}\right)
$$

be its polynomial. We shall apply the following result which is a special example from the theory of algebraic curves. 
(60) There exists a unique formal Laurent series

$$
U(X)=X^{1 / p}+\sum_{h=1}^{\infty} u_{h} X^{-h / p}
$$

with complex coefficients $u_{h}$ such that

$$
F(X, U(X)) \equiv 0 .
$$

Proof. The equation for $U(X)$ has the explicit form

$$
\left(X^{p}-U(X)\right)\left(U(X)^{p}-X\right)=\sum_{r=0}^{p} \sum_{s=0}^{p} E_{r s} X^{r} U(X)^{s},
$$

where again

$$
E_{p p}=E_{p-1, p}=E_{p, p-1}=0
$$

since $h[z]$ is basic.

Similarly as in previous proofs, denote by $U_{h}$ any polynomial in $u_{1}, u_{2}, \cdots, u_{h}$ with complex coefficients; this polynomial may vary in different formulae.

Then evidently

$$
\begin{gathered}
X^{p}-U(X)=X^{p}-X^{1 / p}-\sum_{h=1}^{\infty} u_{h} X^{-h / p}, \\
U(X)^{p}-X=p u_{1} X^{(p-2) / 2}+\sum_{h=2}^{\infty}\left(p u_{h}+U_{h-1}\right) X^{(p-h-1) / p},
\end{gathered}
$$

hence

$$
\left(X^{p}-U(X)\right)\left(U(X)^{p}-X\right)=p u_{1} X^{\left(p^{2}+p-2\right) / p}+\sum_{h=2}^{\infty}\left(p u_{h}+U_{h-1}\right) X^{\left(p^{2}+p-h-1\right) / p} .
$$

On the other hand,

$$
X^{r} U(X)^{s}=X^{(p r+s) / p}+s u_{1} X^{(p r+s-2) / p}+\sum_{h=2}^{\infty}\left(s u_{h}+U_{h-1}\right) X^{(p r+s-h-1) / p} .
$$

Here the sum $p r+s$ for the terms $E_{r s} X^{\prime} U(X)^{s}$ with $E_{r s} \neq 0$ on the right-hand side of (61) does not exceed the value $p^{2}+p-2$ obtained for the pair of suffices $r=p$, $s=p-2$. Hence, on comparing the coefficients of the different powers of $X^{1 / p}$ on the two sides of (61), we obtain the following infinite system of recursive formulae,

$$
p u_{1}=E_{p, p-2}, \quad p u_{h}=U_{h-1} \quad \text { for } \quad h \geqq 2,
$$

which step by step determine all the Laurent coefficients $u_{h}$ uniquely. 
To the result (60) we can add the following corollary.

(62) There exists a positive constant $\Gamma$ such that

$$
\left|u_{h}\right| \leqq \Gamma^{h} \quad(h=1,2,3, \cdots) .
$$

For, if $X$ runs over $C, U(X)$ defines an algebraic function of $X$ in the neighbourhood of a branch point at infinity, and so the Laurent series for $U(X)$ necessarily converges for all sufficiently large finite values of $|X|$.

32. By definition, the algebraic equation

$$
H(X, h[z])=0
$$

has the $p+1$ roots

$$
h\left[z^{p}\right]=z^{-p}+\cdots,
$$

and $h\left[\varepsilon^{j} z^{1 / p}\right]=\varepsilon^{-1} z^{-1 / p}+\cdots$ for $j=0,1, \cdots, p-1$.

On replacing here $X$ by $Y$ and $z$ by $z^{p}$, it follows similarly by the symmetry of $H(X, Y)$ that the equation

$$
H\left(h\left[z^{p}\right], Y\right)=0
$$

has the $p+1$ roots

$$
\begin{gathered}
h\left[z^{p^{2}}\right]=z^{-p^{2}}+\cdots \\
\text { and } h\left[\varepsilon^{j} z\right]=\varepsilon^{-j} z^{-1}+\cdots \text { for } j=0,1, \cdots, p-1
\end{gathered}
$$

On the other hand,

$$
H(X, U(X)) \equiv 0
$$

Here choose

$$
X=h\left[z^{p}\right]=z^{-p}+\cdots,
$$

so that

$$
U(X)=X^{1 / p}+\sum_{h=1}^{\infty} u_{h} X^{-h / p}=z^{-1}+\cdots
$$

becomes a Laurent series in ascending powers of $z$ beginning with the term $z^{-1}$. But $U(X)$ must be one of the series (63), and by the first terms of these series $U(X)$ is necessarily identical with $h[z]$.

It has thus been established that

$$
h[z]=h\left[z^{p}\right]^{1 / p}+\sum_{h=1}^{\infty} u_{h} h\left[z^{p}\right]^{-h / p} .
$$


33. In this identity write

$h\left[z^{p}\right]=z^{-p}(1+s) \quad$ where $\quad s=\sum_{h=1}^{\infty} b_{h} z^{(h+1) p}=b_{1} z^{2 p}+b_{2} z^{3 p}+b_{3} z^{4 p}+\cdots$.

Then for every real number $r$,

$$
h\left[z^{p}\right]^{r}=z^{-p r} \sum_{n=0}^{\infty}\left(\begin{array}{c}
r \\
n
\end{array}\right) s^{n} .
$$

It follows therefore from (64) that

$$
h[z]=z^{-1} \sum_{n=0}^{\infty}\left(\begin{array}{c}
1 / p \\
n
\end{array}\right) s^{n}+\sum_{h=1}^{\infty} \sum_{n=0}^{\infty} u_{h} z^{n}\left(\begin{array}{c}
-h / p \\
n
\end{array}\right) s^{n} .
$$

The left-hand side of this identity is the Laurent series

$$
h[z]=z^{-1}+\sum_{h=1}^{\infty} b_{h} z^{h} .
$$

Also the right-hand side can be written as such a Laurent series. On comparing then again the coefficients of the different powers of $z$, we obtain a new representation of the Laurent coefficients $b_{h}$ of $h[z]$.

It is, however, advisable first to break off both sides of (65) at a suitable finite power of $z$, and for this purpose we introduce the following notation.

Let $M$ and $N$ be any two positive integers. Then firstly, denote by

$$
\left(s^{n} ; M\right)
$$

the polynomial in $z$ which consists of all those terms in the formal power series for $s^{n}$ that involve only the powers $1, z, z^{2}, \cdots, z^{M}$ of $z$. Secondly, let $L_{N}$ be the sum

$$
L_{N}=z^{-1}+\sum_{h=1}^{N} b_{h} z^{h}
$$

of all terms on the left-hand side of (65) that involve only the powers

$$
z^{-1}, z, z^{2}, \cdots, z^{N}
$$

of $z$, and let $R_{N}$ be the analogous sum of terms on the right-hand side of (65). It is obvious that

$$
L_{N}=R_{N}
$$

and it further follows from (65) that

$$
R_{N}=z^{-1}+z^{-1} \sum_{n=1}^{\infty}\left(\begin{array}{c}
1 / p \\
n
\end{array}\right)\left(s^{n} ; N+1\right)+\sum_{h=1}^{\infty} \sum_{n=0}^{\infty} u_{h} z^{h}\left(\begin{array}{c}
-h / p \\
n
\end{array}\right)\left(s^{n} ; N-h\right) .
$$


Here the infinite sums can be replaced by finite ones. For the lowest term in the power series for $s^{n}$ evidently is $b_{1}^{n} z^{2 n p}$, and hence

$$
\left(s^{n} ; M\right)=0 \quad \text { unless } \quad M \geqq 2 n p .
$$

It follows therefore from (68) that also

(69) $R_{N}=z^{-1}+z^{-1} \sum_{n}\left(\begin{array}{c}1 / p \\ n\end{array}\right)\left(s^{n} ; N+1\right)+\sum_{h, n} u_{h} z^{h}\left(\begin{array}{c}-h / p \\ n\end{array}\right)\left(s^{n} ; N-h\right)$

where the sum $\Sigma_{n}$ extends over all integers $n$ satisfying

$$
0 \leqq 2 n p \leqq N+1
$$

and the double sum $\sum_{h . n}$ over all pairs of integers $h, n$ such that

$$
h \geqq 1, \quad n \geqq 0, \quad h+2 n p \leqq N .
$$

34. The numbers $b_{h}$ and $u_{h}$ and the numerical coefficients that occur on the two sides of the equation (67) are real or complex numbers. Denote by the symbol ${ }^{*}$ the operation which replaces all $b_{h}$ and $u_{h}$ and all these coefficients by their absolute values and which simultaneously substitute 1 for $z$. If * changes $L_{N}$ and $R_{N}$ into $L_{N}^{*}$ and $R_{N}^{*}$, respectively, it is obvious that

$$
L_{N}^{*}=1+\sum_{h=1}^{N}\left|b_{h}\right|
$$

and

$$
L_{N}^{*} \leqq R_{N}^{*}
$$

An upper estimate for $R_{N}^{*}$ can be obtained as follows. Denote by

$$
\left[s^{n} ; M\right]
$$

the result of the operation ${ }^{*}$ applied to $\left(s^{n} ; M\right)$. By the definition of $s$,

$$
s^{n}=\sum_{h_{1}=1}^{\infty} \cdots \sum_{h_{n}=1}^{\infty} b_{h_{1}} \cdots b_{h_{n}} z^{p\left(h_{1}+\cdots+h_{n}+n\right)},
$$

whence

$$
\left[s^{n} ; M\right]=\sum_{n_{1}, \cdots, h_{n}}\left|b_{h_{1}} \cdots b_{h_{n}}\right|
$$

where the multiple summation is extended over all sets of $n$ positive integers $h_{1}, \cdots, h_{n}$ satisfying

$$
p\left(h_{1}+\cdots+h_{n}+n\right) \leqq M .
$$

The number of terms on the right-hand side of (72) is therefore equal to 


$$
\left(\begin{array}{c}
{[M / p]} \\
n
\end{array}\right) \leqq 2^{M / p}
$$

and the suffices $h_{1}, \cdots, h_{n}$ are all less than $M / p$.

We know already that

$$
\left|u_{h}\right| \leqq \Gamma^{h} \quad(h=1,2,3, \cdots) .
$$

Assume that there further exists a constant $C$ satisfying

$$
C \geqq 5 \text { and } C \geqq 2^{p-1} \Gamma^{p}+1
$$

such that

$$
\left|b_{h}\right| \leqq C^{h} \quad \text { for } \quad 1 \leqq h \leqq N-1,
$$

where $N$ is a certain integer not less than 6 . For $N=6$ this assumption is certainly admissible.

35. Let

$$
M \leqq p N,
$$

an inequality which holds in particular when $M=N+1$. The suffices $h_{1}, \cdots, h_{n}$ in (72) are smaller than $M / p$ and hence cannot exceed $N-1$. The hypothesis (74) may therefore be applied to all the coefficients $b_{h}$ in the multiple sum (72).

Since this sum has at most $2^{M / p}$ terms, and since $h_{1}+\cdots+h_{n}$ is for all these terms at most $(M / p)-n$, it follows that

$$
\left[s^{n} ; M\right] \leqq 2^{M / p} \cdot C^{(M / p)-n} \text { for } 1 \leqq M \leqq p N .
$$

By the definition of $R_{N}^{*}$ the equation (69) implies then the upper estimate

$$
\begin{aligned}
R_{N}^{*} \leqq 1 & +\sum_{n}\left|\left(\begin{array}{c}
1 / p \\
n
\end{array}\right)\right| 2^{(N+1) / p} \cdot C^{((N+1) / p)-n}+ \\
& +\sum_{h, n} \Gamma^{h}\left|\left(\begin{array}{c}
-h / p \\
n
\end{array}\right)\right| 2^{(N-h) / p} \cdot C^{((N-h) / p)-n} .
\end{aligned}
$$

Here the sums $\Sigma_{n}$ and $\Sigma_{h, n}$ are defined as in $\$ 33$.

In the first sum,

$$
\left|\left(\begin{array}{c}
1 / p \\
n
\end{array}\right)\right| \leqq 1 \text { for all } n, \text { and } \sum_{n=0}^{\infty} C^{-n}=\frac{C}{C-1}<2
$$

by the first assumption (73). Hence

$$
\sum_{n}\left|\left(\begin{array}{c}
1 / p \\
n
\end{array}\right)\right| 2^{(N+1) / p} \cdot C^{((N+1) / p)-n} \leqq(2 C)^{(N+1) / p} \sum_{n=0}^{\infty} C^{-n}<2(2 C)^{(N+1) / p}
$$


In the second sum,

$$
\left(\begin{array}{c}
-h / p \\
n
\end{array}\right) \text { has the same sign as }(-1)^{n},
$$

so that

$$
\sum_{n=0}^{\infty}\left|\left(\begin{array}{c}
-h / p \\
n
\end{array}\right)\right| C^{-n}=\sum_{n=0}^{\infty}\left(\begin{array}{c}
-h / p \\
n
\end{array}\right)(-C)^{-n}=\left(1-C^{-1}\right)^{-h / p} .
$$

Therefore, by the second assumption (73),

$$
\sum_{h, n} \Gamma\left|\left(\begin{array}{c}
-h / p \\
n
\end{array}\right)\right| 2^{(N-h) / p} \cdot C^{((N-h) / p)-n} \leqq \sum_{h=1}^{\infty} \Gamma^{h}\left(1-C^{-1}\right)^{-h / p} \cdot(2 C)^{(N-h) / p}=
$$

$$
=(2 C)^{N / p} \sum_{h=1}^{\infty}\left(\Gamma^{-p} \cdot 2(C-1)\right)^{-h / p}<(2 C)^{N / p} \sum_{h=1}^{\infty} 2^{-h}=(2 C)^{N / p} .
$$

On combining the two upper estimates (75) and (76) with the earlier formulae (70) and (71), it follows finally that

$$
L_{N}^{*}=1+\sum_{h=1}^{N}\left|b_{h}\right| \leqq R_{N}^{*} \leqq 1+2(2 C)^{(N+1) / p}+(2 C)^{N / p}<1+3(2 C)^{(N+1) / p},
$$

whence, in particular,

$$
\left|b_{N}\right|<3(2 C)^{(N+1) / p} .
$$

36. This estimate can finally be replaced by

$$
\left|b_{N}\right|<C^{N}
$$

provided it can be established that

$$
3(2 C)^{(N+1) / p} \leqq C^{N} .
$$

This inequality will be satisfied if

$$
3 \times 2^{(N+1) / p} \leqq C^{N-(N+1) / p},
$$

or equivalently, if

$$
C \geqq 3^{p[(p-1) N-\mathrm{t}]^{-1}} \times 2^{(N+1)[(p-1) N-1]^{-1}} .
$$

This formula is, in fact, true. For $N \geqq 6$ and $p \geqq 2$, hence

$$
p[(p-1) N-1]^{-1} \leqq 1 / 2 \text { and }(N+1)[(p-1) N-1]^{-1} \leqq 3 / 2,
$$

and further

$$
3^{1 / 2} \times 2^{3 / 2}=24^{1 / 2}<5,
$$

while, on the other hand, $C$ is at least 5 by the first assumption (73). 
The inequality (78) is therefore valid. Hence the induction hypothesis (74), which was assumed to be true for $N$, holds also for $N+1$. As it could be assumed for $N=6$, it holds then for every positive integer $N$, and so

$$
\left|b_{h}\right| \leqq C^{h} \quad(h=1,2,3, \cdots) .
$$

37. These inequalities (79) mean that the formal Laurent series $h[z]$ is in fact convergent for

$$
0<|z|<1 / C
$$

In other words, $h[z]$ is a single-valued analytic function for $|z|<1 / C$ with a simple pole of residue 1 at $z=0$.

This result has been proved for every formal basic $S_{p}$-series with complex coefficients. It can immediately be generalised as follows.

THEOREM 8. Let $f(z)$ be an arbitrary formal $S_{p}$-series over $C$. Then there exists a positive constant $\gamma$ such that $f(z)$ converges for $0<|z|<\gamma$ and hence represents a single-valued analytic function for $|z|<\gamma$. The origin $z=0$ is a pole of $f(z)$ if $f(z)$ is of negative order, but is a regular point if the order of $f(z)$ is non-negative.

PROOF. If the order $m$ of $f(z)$ is negative, then by $\S 15$ there exist a basic $S_{p}$-series $h[z]$ and two constants $C_{0} \neq 0$ and $C_{1}$ such that $f(z)=C_{0} h\left[z^{-m}\right]+C_{1}$. If the order $m$ of $f(z)$ is positive, then Theorem 3 shows that $f(z)^{-1}$ is an $S_{p}$-series of the negative order $-m$. Hence in this case $f(z)$ itself can be written in the form

$$
f(z)=\left(C_{0} h\left[z^{m}\right]+C_{1}\right)^{-1} .
$$

If, finally, $f(z)$ has the order 0 and $a_{0}$ is its constant term, then $f(z)-a_{0}$ is an $S_{\mathrm{p}}$-series of a certain positive order $m$, and so $f(z)$ allows a representation

$$
f(z)=\left(C_{0} h\left[z^{m}\right]+C_{1}\right)^{-1}+a_{0} .
$$

Our general convergence result for basic $S_{p}$-series implies then the assertion since in the last two cases $f(z)$ can be expressed as a power series convergent in a neighbourhood of $z=0$.

38. If $f(z)$ is any $S_{p}$-series over $C$, then the same symbol $f(z)$ is used to denote the analytic function which is defined by the series in its region of convergence is from here extended into its whole domain of existence by analytic continuation. Such an analytic function is called an $S_{p}$-function. In the special case when $f(z)=h[z]$ is a basic $S_{p}$-series, we speak of basic $S_{p}$-functions. It would evidently suffice to study basic $S_{p}$-functions. 
There are basic $S_{p}$-functions which exist and are single-valued in the whole $z$-plane; for instance, the rational functions $z^{-1}$ and $z^{-1}+z$ are for every prime $p$ of this kind. I do not know whether there are also non-rational algebraic basic $S_{p}$-functions. On the other hand, I conjecture that there exist transcendental basic $S_{p}$-functions which can be continued into the whole $z$-plane, but may be multi-valued.

There do exist basic $S_{p}$-functions which can be defined only in the unit circle

$$
U:|z|<1,
$$

and which in $U$ are regular and single-valued except for the pole at $z=0$. A particularly important example of this kind of function can be derived from the modular function

$$
j(\omega)=\sum_{n=-1}^{\infty} a_{h} e^{2 p i \omega} \quad\left(a_{-1}=1, a_{0}=744, \text { etc. }\right)
$$

by putting

$$
z=e^{2 \pi i \omega}
$$

and subtracting from $j(\omega)$ its constant term $a_{0}=744$. The transformation equations for $j(\omega)$ imply that the derived Laurent series

$$
g[z]=z^{-1}+\sum_{h=1}^{\infty} a_{h} z^{h}
$$

defines for every prime $p$ a basic $S_{p}$-function. Since $j(\omega)$ exists only in the complex upper halfplane, $g[z]$ is defined in $U$, but has the circle $|z|=1$ as its natural boundary. It is single-valued and regular in $U$ except for its pole at $z=0$.

39. For arbitrary $S_{p}$-functions only a weaker result can be proved.

THEOREM 9. Every basic $S_{p}$-function can be continued into the whole of $U$, but may become multi-valued by having a certain set of branch points in $U$, all of finite orders. The function is regular at every point distinct from its pole $z=0$ and from these branch points.

The same result holds for general $S_{p}$-functions of negative orders. For general $S_{p}$-functions of non-negative orders the same result remains valid except that now there is no pole at $z=0$, but there may be poles elsewhere.

Proof. It evidently suffices to prove the assertion for basic $S_{p}$-functions $h[z]$. In this case we know already that the only singularity of $h[z]$ in a certain region $|z|<1 / C$ is the pole at $z=0$.

Let the assertion be false. There exists then a largest open disc 
where $0<\rho<1$, in which the assertion is still true. We shall prove that this assumption leads to a contradiction by constructing a larger open disc in which $h[z]$ has still the same properties.

For this purpose denote by $\rho^{\prime}$ a constant satisfying

$$
\rho^{\prime p}<\rho<\rho^{\prime}<1,
$$

and let then $V_{1}$ and $V_{2}$ be the discs

$$
V_{1}:|z| \leqq \rho^{\prime p} \quad \text { and } \quad V_{2}:|z| \leqq \rho^{\prime},
$$

respectively. Thus $V_{1}$ is contained in $V$, and $V$ is contained in $V_{2}$.

If $H(X, Y)$ is again the polynomial of $h[z]$, denote by $A(X)$ the algebraic function of the complex variable $X$ which is defined by

$$
H(X, A(X))=0 .
$$

(The formal Laurent series $U(X)$ of $\$ 31$ defines thus one of the branches of $A(X)$ at the point of infinity.) Since $H(X, Y)$ is monic in $X, A(X)$ is regular for finite $X$ except at its branch points, but also these branch points are not poles of $A(X)$.

We know that

$$
H\left(h[z], h\left[z^{p}\right]\right)=0
$$

identically in $z$. The definition of $A(X)$ implies therefore that

$$
h[z]=A\left(h\left[z^{p}\right]\right) \text {. }
$$

Let now $z$ run over the closed disc $V_{2}$ so that $z^{p}$ runs over the closed disc $V_{1}$ and hence lies in a closed subset of $V$. By the hypothesis about $V$ the only singularities of $h\left[z^{p}\right]$ are then its pole at $z=0$ and a certain set of branch points of finite orders; here this set can consist at most of finitely many branch points. Since $h[z]$ is an algebraic function of $h\left[z^{p}\right]$, it has the same properties for $z$ in $V_{2}$. But by the definition of $\rho^{\prime}, V$ is a proper subset of the interior $|z|<\rho^{\prime}$ of $V_{2}$. This implies our assertion and proves the theorem.

40. For the special case when $p=2$ and $H(X, Y)$ is reducible, we shall now construct a basic $S_{p}$-function $h[z]$ which is multi-valued and in fact has infinitely many branch points in $U$.

By the remark to the formula (37) of $\$ 20$ the polynomial $H(X, Y)$ must have the form

$$
H(X, Y)=-\left(X^{2}-Y+c\right)\left(Y^{2}-X+c\right) ;
$$

here $c$ lies in $C$. For simplicity, let $c$ be a positive constant. 
In the present case the basic function

$$
h[z]=z^{-1}+\sum_{h=1}^{\infty} b_{h} z^{h}
$$

satisfies the functional equation

$$
h[z]^{2}-h\left[z^{2}\right]+c=0
$$

from which it easily follows that $h[z]$ is an odd function, hence that

$$
b_{2 h}=0 \text { for } h=1,2,3, \cdots \text {. }
$$

We further find by the usual method that

$$
b_{1}=-c / 2 \text { and } 2 b_{2 h+1}=-\sum_{j=0}^{h-1} b_{2 j+1} b_{2 h-2 i-1}+b_{h} \text { for } h \geqq 1 .
$$

These formulae show in particular that all the coefficients $b_{2 h+1}$ are negative. Thus, on taking only the term with $j=0$ in the sum for $2 b_{2 h+1}$, it follows that

$$
2 b_{2 h+1} \leqq-b_{1} b_{2 h-1} \quad \text { if } \quad h \geqq 1
$$

and therefore that

$$
b_{2 h+1} \leqq-(c / 4)^{h} \quad(h=0,1,2, \cdots) .
$$

Hence, if $z$ is positive and the left-hand series converges,

$$
\sum_{h=1}^{\infty} b_{h} z^{h}=\sum_{h=0}^{\infty} b_{2 h+1} z^{2 h+1} \leqq-2 z \sum_{h=0}^{\infty}\left(c z^{2} / 4\right)^{h}=-8 z\left(4-c z^{2}\right)^{-1} .
$$

Denote by $\gamma$ the radius of convergence of $\sum_{h=1}^{\infty} b_{h} z^{h}$. This estimate for the series, and the fact that $\gamma$ is positive by Theorem 8 , imply that

$$
0<\gamma<2 c^{-1 / 2} \text {. }
$$

Choose therefore $c>4$; then

$$
0<\gamma<1 \text {. }
$$

Since all the coefficients $b_{h}$ are negative or zero, the point $z=\gamma$ on the positive real axis is a singular point of $h[z]$, but by $\gamma^{2}<\gamma$ naturally is a regular point of $h\left[z^{2}\right]$. By Theorem $9, z=\gamma$ necessarily is a branch point of $h[z]$. The functional equation requires then that

$$
h\left[\gamma^{2}\right]=c .
$$

Therefore $h\left[z^{2}\right]-c$ has a convergent development of the form

$$
h\left[z^{2}\right]-c=\sum_{h=1}^{\infty} c_{h}^{\prime}(z-\gamma)^{h}
$$


where $c_{1}^{\prime} \neq 0$, in a certain neighbourhood of $z=\gamma$. Hence, by the functional equation, $h[z]$ allows in a possibly smaller neighbourhood of $z=\gamma$ a convergent development

$$
h[z]=\sum_{h=1}^{\infty} c_{h}(z-\gamma)^{h / 2}
$$

where $c_{1} \neq 0$.

On applying now the functional equation repeatedly, we see that also the infinitely many points

$$
e^{2 \pi i m \cdot 2^{-n}} \gamma^{2-n} \quad\left(\begin{array}{c}
m=0,1, \cdots, 2^{n}-1 \\
n=0,1,2, \cdots
\end{array}\right)
$$

are branch points of $h[z]$, possibly of higher order. These branch points converge to every point of $|z|=1$. This circle forms therefore the natural boundary of $h[z]$.

The same proof leads to the following result.

Theorem 10. A basic $S_{p}$-series $h[z]$ defines a basic $S_{p}$-function with at least one branch point inside the unit circle if and only if its radius of convergence is less than 1 .

For there is again at least one singular point of $h[z]$ on the circle of convergence, and if its radius is less than 1 , then by Theorem 9 this singular point must be a branch point.

Just as in the example, if $h[z]$ has at least one branch point inside $|z|=1$, it probably has an infinite sequence of branch points tending to this circle which therefore becomes again a natural boundary.

Unfortunately, the actual determination of the radius of convergence of a general basic $S_{p}$-series $h[z]$ when its polynomial $H(X, Y)$ is given does not seem to be an easy problem.

\section{Chapter 4. Modular functions and $S_{p}$-functions}

41. In this final chapter we shall study relations between the theory of $S_{p}$-functions and that of certain modular functions.

To begin with, let

$$
j(\omega)=\sum_{h=-1}^{\infty} a_{h} z^{2 \pi i h \omega} \quad\left(a_{-1}=1\right)
$$

be the modular function of level 1. Put

$$
z=e^{2 \pi i \omega}
$$


and

$$
\begin{gathered}
f(z)=z^{-1}+\sum_{h=0}^{\infty} a_{h} z^{h} \text { and } h[z]=z^{-1}+\sum_{h=1}^{\infty} a_{h} z^{h}, \\
\text { so that } f(z)=h[z]+a_{0} .
\end{gathered}
$$

Hence $f(z)$ is a normed $S_{p}$-function of order -1 , and $h[z]$ is a basic $S_{p}$-function, both for every prime $p$. Both functions are regular for $|z|<1$, but have the circle $|z|=1$ as their natural boundaries; the origin is a simple pole of $f(z)$ and $h[z]$.

One has computed the coefficients $a_{h}$ for all suffices $h \leqq 500$. Here are a few of the first coefficients.

$$
\begin{array}{ll}
a_{0}=744 & a_{7}=4465,69940,71935 \\
a_{1}=1,96884 & a_{8}=40149,08866,56000 \\
a_{2}=214,93760 & a_{9}=3,17644,02297,84420 \\
a_{3}=8642,99970 & a_{10}=22,56739,33095,93600 \\
a_{4}=2,02458,56256 & a_{11}=146,21191,14995,19294 \\
a_{5}=33,32026,40600 & a_{12}=874,31371,96857,75360 \\
a_{6}=425,20233,00096 &
\end{array}
$$

For every prime $p$, the function $j(\omega)$ satisfies the transformation equation

$$
F_{p}(j(p \omega), j(\omega))=0,
$$

where $F_{p}(X, Y)$ is a symmetric polynomial of degree $p+1$ in $X$ and $Y$ which is monic in both. Hence $f(z)$ satisfies the functional equation

$$
F_{p}\left(f\left(z^{p}\right), f(z)\right)=0
$$

and is by Theorem 1 a normed $S_{p}$-function with the polynomial $F_{p}(X, Y)$. Similarly, $h[z]$ is a basic $S_{p}$-function.

Since we may substitute the $a_{h}$ for the $b_{h}$, the coefficients $a_{h}$ satisfy for every prime $p$ the corresponding infinite system of recursive formulae which allows successively to determine all the coefficients with $h \geqq p^{2}+p$ as polynomials in these coefficients with $1 \leqq h \leqq p^{2}+p-1$; in addition, the latter satisfy $[p(p-1)] / 2$ conditions.

For the lowest cases, we found for $p=2$ the recursive formulae (46) together with the one condition (44), and for $p=3$ the recursive formulae (51) together with three conditions (48). For the next two cases $p=5$ and $p=7$ we determined only the recursive formulae for the coefficients with suffices $5 h$ and $7 h$, respectively, as well as a few other coefficients. 
Included in these recursive formulae and conditions are in particular the following simple equations,

$$
\begin{array}{ll}
a_{4}=a_{3}+\left(a_{1}^{2}-a_{1}\right) / 2, & a_{9}=a_{5}+a_{1} a_{3}+a_{2}^{2}+\left(a_{1}^{3}-a_{1}\right) / 3, \\
a_{6}=a_{4}+a_{1} a_{2}, & a_{10}=a_{8}+a_{1} a_{4}+a_{2} a_{3}, \\
a_{8}=a_{5}+a_{1} a_{3}+\left(a_{2}^{2}-a_{2}\right) / 2, & a_{12}=a_{6}+a_{1} a_{4}+2 a_{2} a_{3}+a_{1}^{2} a_{2} .
\end{array}
$$

From the earlier table of the numerical values of the coefficients they can easily be checked.

It needs scarcely be stated that the recursive formulae for $p=2$ are useful for the computation of the coefficients $a_{h}$ of $j(\omega)$, i.e., of $h[z]$.

42. Denote by $K(p)$ the set of all recursive formulae and conditions that hold for the prime $p$ and for every basic $S_{p}$-function $h[z]$. The function $h[z]$ derived from $j(\omega)$ is an $S_{p}$-function for every prime $p$ and is basic, hence satisfies $K(p)$ for every prime $p$. Therefore the infinitely many sets of formulae

$$
K(2), K(3), K(5), K(7), \cdots
$$

all hold for this special function $h[z]$ and so are consistent with one another.

Our original investigation of basic $S_{p}$-series and $S_{p}$-functions involved only one set $K(p)$ belonging to a single prime $p$. It is then rather surprising that the existence of the modular function $j(\omega)$ and its property of satisfying all the transformation equations of prime orders should imply certain relations between the different sets $K(p)$. It would have great interest to study these relations.

43. The theory of $S_{p}$-functions allows to prove two theorems that explain in how far the modular function $j(\omega)$ is determined by its transformation equations of any prime order.

THEOREM 11. Let $p$ be a prime and $F_{p}(X, Y)$ the transformation polynomial of $j(\omega)$ of order $p$. Let further

$$
\phi(z)=z^{-1}+\sum_{h=0}^{\infty} \alpha_{h} z^{h}
$$

be a formal Laurent series with coefficients in $C$ such that

$$
F_{p}\left(\phi\left(z^{p}\right), \phi(z)\right)=0 .
$$

Then $\phi(z)$ converges and defines a single-valued regular function for $0<|z|<1$, and

$$
j(\omega)=\phi\left(e^{2 \pi i \omega)}\right.
$$

identically for all $\omega$ in the complex upper halfplane. 
Proof. The polynomial $F_{p}(X, Y)$ is symmetric, monic, and of the exact degree $p+1$ in $X$ and $Y$. Further the formal series

$$
\phi\left(z^{p}\right), \text { and } \phi\left(\varepsilon^{j} z^{1 / p}\right) \text { where } j=0,1, \cdots, p-1 \text {, }
$$

obviously are all distinct. It follows then from Theorem 1 that $\phi(z)$ is a normed $S_{p}$-series of order -1 , and that $F_{p}(X, Y)$ is its polynomial. But the series $f(z)$ defined in $\$ 41$ had exactly the same properties. Hence, by $\S 12$ and by Theorem 4 , the two series $\phi(z)$ and $f(z)$ are identical, whence the assertion.

THEOREM 12. Let $p$ be a prime, and let

$$
\phi(z)=z^{-1}+\sum_{h=0}^{\infty} \alpha_{h} z^{h}
$$

be a formal $S_{p}$-series such that

$$
\alpha_{h}=a_{h} \quad \text { for } \quad 0 \leqq h \leqq p^{2}+p-1,
$$

where the $a_{h}$ are the Fourier coefficients of $j(\omega)$ as defined in $\$ 41$. Then $\phi(z)$ converges and defines a single-valued function for $0<|z|<1$, and

$$
j(\omega)=\phi\left(e^{2 \pi i \omega}\right)
$$

identically for all $\omega$ in the complex upper halfplane.

Proof. The coefficients $\alpha_{h}$ of $f(z)$ coincide for $0 \leqq h \leqq p^{2}+p-1$, from which it follows that the polynomials $\Phi(X, Y)$ of $\phi(z)$ and $F_{p}(X, Y)$ of $f(z)$ are identical. For both polynomials are determined uniquely by the same set of coefficients $\alpha_{h}=a_{h}$ with $0 \leqq h \leqq p^{2}+p-1$. The assertion is therefore contained in Theorem 11.

A completely different characterisation of $j(\omega)$ by its transformation equations was given by Siegel, (1964). It depended on the behaviour of $j(\omega)$ near fixed points of elliptic transformations.

44. There are modular functions of higher level which for suitable primes $p$ lead to $S_{p}$-functions.

As a first example consider the cube root of $j(\omega)$. In the notation of $\mathrm{H}$. Weber, (1908), p. 179, put

$$
\gamma_{2}(\omega)=j(\omega)^{1 / 3}=h[z]=z^{-1}+\sum_{k=0}^{\infty} b_{3 k+2} z^{3 k+2},
$$

where now, in the series for $h[z], z$ denotes the cube root of the previous $z$,

$$
z=e^{2 \pi i \omega / 3}
$$

and where the first coefficients $b_{3 k+2}$ have the values 


$$
b_{2}=248, \quad b_{5}=4124, \quad b_{8}=34752 .
$$

By Weber, $(1908$, l.c. p. 248$), h[z]$ certainly is a basic $S_{2}$-function. For, as he shows, the three functions $h\left[z^{2}\right], h\left[z^{1 / 2}\right]$, and $h\left[-z^{1 / 2}\right]$ are roots of

$$
F(X, h[z])=0,
$$

where $F(X, Y)$ in the present case denotes the symmetrical polynomial

$$
F(X, Y)=-\left(X^{2}-Y\right)\left(Y^{2}-X\right)+496 X Y-54000=0 .
$$

Since $h[z]$ is an $S_{2}$-function, its coefficients $b_{h}$ must satisfy the former condition (44) and the recursive formulae (46) of $\$ \$ 24-25$. Here (44) trivially is valid since $b_{h}$ always vanishes when $h \neq 2(\bmod 3)$. For the same reason, the recursive formulae (46) take now the following simpler form:

$$
\begin{gathered}
b_{12 k+2}=b_{6 k+2}+\sum_{j=0}^{k-1} b_{3 j+2} b_{6 k-3 j-1} \\
b_{12 k+5}=b_{6 k+5}+\sum_{j=0}^{k-1} b_{3 j+2} b_{6 k-3 j+2}-\sum_{j=0}^{2 k-1}(-1)^{j-1} b_{3 j+2} b_{12 k-3 j+2}+ \\
+\sum_{j=0}^{k-1} b_{3 j+2} b_{12 k-12 j-4}-b_{2} b_{6 k+2}+\left(b_{3 k+2}^{2}-b_{3 k+2}\right) / 2+ \\
+\left(b_{6 k+2}^{2}+b_{6 k+2}\right) / 2 \\
b_{12 k+8}=b_{6 k+5}+\sum_{j=0}^{k-1} b_{3 j+2} b_{6 k-3 j+2}+\left(b_{3 k+2}^{2}-b_{3 k+2}\right) / 2 \\
b_{12 k+11}=b_{6 k+8}+\sum_{j=0}^{k} b_{3 j+2} b_{6 k-3 j+5}-\sum_{j=0}^{2 k}(-1)^{j-1} b_{3 j+2} b_{12 k-3 j+8}+ \\
+\sum_{j=0}^{k} b_{3 j+2} b_{12 k-12 j+2}-b_{2} b_{6 k+5}-\left(b_{6 k+5}^{2}-b_{6 k+5}\right) / 2
\end{gathered}
$$

Here as usual empty sums mean 0 , and $k$ may be any non-negative integer.

These recursive formulae can again be used to evaluate any number of coefficients $b_{3 k+2}$ of $h[z]$. For the lowest suffices,

$$
b_{11}=2,13126, \quad b_{14}=10,57504, \quad b_{17}=45,30744, \quad b_{20}=173,33248 \text {. }
$$

It would be interesting to decide whether $h[z]$ is an $S_{p}$-function for all primes $p \equiv 2(\bmod 3)$. The function in fact has this property when $p=5$, as follows from the form of the transformation equation between $j(\omega)$ and $j(5 \omega)$ as given in R. Fricke, (1922), p. 393.

45. As a second example consider the Jacobi module 
$u(\omega)=k^{1 / 4}=2^{1 / 2} q^{1 / 8} \prod_{m=1}^{\infty} \frac{1+q^{2 m}}{1+q^{2 m-1}}=$

where

$$
=2^{1 / 2} q^{1 / 8}\left(1-q+2 q^{2}-3 q^{3}+4 q^{4}-6 q^{5}+9 q^{6}-\cdots\right),
$$

$$
q=e^{\pi i \omega}
$$

Put

so that

$$
z=e^{\pi i \omega / 8} \text { and } h[z]=(4 / k)^{1 / 4}
$$

$$
h[z]=z^{-1} \prod_{m=1}^{\infty} \frac{1+z^{16 m-8}}{1+z^{16 m}}=z^{-1}+\sum_{k=0}^{\infty} b_{8 k+7} z^{8 k+7}
$$

where the lowest coefficients are

$$
b_{7}=b_{63}=+1, \quad b_{15}=b_{47}=b_{55}=b_{79}=-1, \quad b_{23}=b_{31}=b_{39}=b_{71}=0 .
$$

Let now $p$ be a prime satisfying

$$
p \equiv \pm 1 \quad(\bmod 8)
$$

Then $v(\omega)=u(p \omega)$ is connected with $u(\omega)$ by a symmetric transformation equation which in the lowest case $p=7$ has the form (Fricke, l.c., p. 501)

$$
u^{8}+v^{8}-u v\left(8 u^{6} v^{6}-28 u^{5} v^{5}+56 u^{4} v^{4}-70 u^{3} v^{3}+56 u^{2} v^{2}-28 u v+8\right)=0 .
$$

It follows from Theorem 1 that $u$ is an $R_{7}$-function of order +1 . By $\$ 9$, this explains why in this equation all terms different from $u^{7}+v^{7}$ are divisible by $u v$.

We deduce by a trivial change of variables in this equation that the polynomial $H(X, Y)$ of $h[z]$ is given by

$$
\begin{aligned}
F(X, Y)=-\left(X^{7}-Y\right)\left(Y^{7}-X\right)+ \\
\quad+7\left(X^{6} Y^{6}-4 X^{5} Y^{5}+10 X^{4} Y^{4}-16 X^{3} Y^{3}+16 X^{2} Y^{2}-9 X Y\right)=0 .
\end{aligned}
$$

There are thus particularly simple explicit expressions as polynomials in $h[z]$ for the elementary symmetric functions $s_{1}, s_{2}, \cdots, s_{8}$ of the elements of $\Sigma_{h}$. These would allow again to derive recursive formulae for the coefficients $b_{8 k+7}$.

It is clear that $h[z]$ is a basic $R_{p}$-function for all the primes $8 n \pm 1$.

46. As a final example consider the Schlaefli modular equations which concern the function (Fricke, l.c., pp. 502-8)

$$
\begin{aligned}
s(\omega) & =2^{1 / 3}\left(k k^{\prime}\right)^{1 / 12}=2^{1 / 2} q^{1 / 24} \prod_{n=1}^{\infty}\left(1+q^{2 n-1}\right)^{-1}= \\
& =2^{1 / 2} q^{1 / 24}\left(1-q+q^{2}-2 q^{3}+2 q^{4}-3 q^{5}+4 q^{6}-\cdots\right),
\end{aligned}
$$


where again $q=e^{\pi i \omega}$. Put now

$$
z=e^{\pi i \omega / 24} \text { and } h[z]=2^{1 / 2} s(\omega)^{-1},
$$

so that

$$
h[z]=q^{-1 / 24} \prod_{n=0}^{\infty}\left(1+q^{2 n+1}\right)=z^{-1}+\sum_{k=0}^{\infty} b_{24 k+23} z^{24 k+23},
$$

with the lowest coefficients

$$
\begin{gathered}
b_{23}=b_{71}=b_{95}=b_{119}=b_{143}=b_{167}=+1, \quad b_{47}=0, \\
b_{191}=b_{215}=b_{239}=b_{263}=+2 .
\end{gathered}
$$

Denote by $p$ any prime not less than 5 . Then the functions $s(\omega)$ and $t(\omega)=s(p \omega)$ are connected by a symmetric algebraic equation with the highest terms $s^{p+1}$ and $t^{p+1}$. Hence Theorem 1 leads again easily to the result that in the present case $h[z]$ is a basic $S_{p}$-function (and even a basic $\boldsymbol{R}_{p}$-function) for all such primes.

For the lowest primes $p=5$ and $p=7$ the transformation equations are

$$
s^{6}+t^{6}+s t\left(s^{4} t^{4}-4\right)=0 \text { and } s^{8}+t^{8}-s t\left(s^{6} t^{6}-7 s^{3} t^{3}+8\right)=0,
$$

respectively. This means that for $p=5$ the polynomial $H(X, Y)$ of $h[z]$ is equal to

$$
H(X, Y)=-\left(X^{5}-Y\right)\left(Y^{5}-X\right)+5 X Y
$$

and for $p=7$ equal to

$$
H(X, Y)=-\left(X^{7}-Y\right)\left(Y^{7}-X\right)+7\left(X^{4} Y^{4}-X Y\right) .
$$

Of particular interest is the simple result for $p=5$. From the explicit form of $H(X, Y)$ the elementary symmetric functions of the elements of $\Sigma_{h}$ are given by

$$
s_{1}=h[z]^{5}, \quad s_{2}=s_{3}=s_{4}=0, \quad s_{5}=-h[z], \quad s_{6}=h[z]^{6} .
$$

By means of these formulae it would again not be difficult, but rather tedious, to derive recursive formulae for the coefficients $b_{24 k+23}$. But since evidently $b_{24 k+23}$ is equal to the number of partitions of $k+1$ into distinct odd integers, there is no need to do so.

\section{References}

R. Fricke, (1922), Elliptische Funktionen, Teil 2.

K. Mahler, (1929), 'Arithmetische Eigenschaften der Lösungen einer Klasse von Funktionalgleichungen', Math. Annalen 101, 342-366. 
K. Mahler, (1930a), 'Über das Verschwinden von Potenzreihen mehrerer Veränderlichen in speziellen Punktfolgen', Math. Annalen, 103, 573-587.

K. Mahler, (1930b), 'Arithmetische Eigenschaften einer Klasse transzendental-transzendenter Funktionen', Math. Z. 32, 545-585.

C. L. Siegel, (1964), 'Bestimmung der elliptischen Modulfunktion durch eine Transformationsgleichung', Abh. Sem. Univ. Hamburg 27, 32-38.

H. Weber, (1908), Lehrbuch der Algebra, Band 3.

Department of Mathematics

Research School of Physical Sciences

Australian National University

Canberra, A.C.T. 2600

Australia. 